\title{
Supersymmetric dark matter in the light of CERN LEP and the Fermilab Tevatron collider
}

\author{
John Ellis, ${ }^{1}$ Toby Falk, ${ }^{2}$ Gerardo Ganis, ${ }^{3}$ and Keith A. Olive ${ }^{4}$ \\ ${ }^{1}$ TH Division, CERN, Geneva, Switzerland \\ ${ }^{2}$ Department of Physics, University of Wisconsin, Madison, Wisconsin 53706 \\ ${ }^{3}$ Max-Planck-Institut für Physik, Munich, Germany \\ ${ }^{4}$ Theoretical Physics Institute, School of Physics and Astronomy, University of Minnesota, Minneapolis, Minnesota 55455
}

(Received 20 April 2000; published 12 September 2000)

\begin{abstract}
We analyze the accelerator constraints on the parameter space of the minimal supersymmetric extension of the standard model, comparing those now available from CERN LEP II and anticipating the likely sensitivity of Fermilab Tevatron run II. The most important limits are those from searches for charginos $\chi^{ \pm}$, neutralinos $\chi_{i}$ and Higgs bosons at LEP, and searches for top squarks, charginos and neutralinos at the Tevatron collider. We also incorporate the constraints derived from $b \rightarrow s \gamma$ decay, and discuss the relevance of charge- and color-breaking minima in the effective potential. We combine and compare the different constraints on the Higgs-mixing parameter $\mu$, the gaugino-mass parameter $m_{1 / 2}$ and the scalar-mass parameter $m_{0}$, incorporating radiative corrections to the physical particle masses. We focus on the resulting limitations on supersymmetric dark matter, assumed to be the lightest neutralino $\chi$, incorporating coannihilation effects in the calculation of the relic abundance. We find that $m_{\chi}>51 \mathrm{GeV}$ and $\tan \beta>2.2$ if all soft supersymmetry-breaking scalar masses are universal, including those of the Higgs bosons, and that these limits weaken to $m_{\chi}>46 \mathrm{GeV}$ and $\tan \beta>1.9$ if nonuniversal scalar masses are allowed. Light neutralino dark matter cannot be primarily Higgsino in composition.
\end{abstract}

PACS number(s): 12.60.Jv, 14.80.Ly, 95.35.+d

\section{INTRODUCTION}

The search for experimental evidence for supersymmetry is currently approaching a transition. For several years now, many of the most incisive experimental searches have been those at the CERN $e^{+} e^{-}$collider LEP [1], whose constraints on the parameter space of the minimal supersymmetric extension of the standard model (MSSM) have grown ever more restrictive, as the center-of-mass energy of LEP II has been increased in successive steps. In parallel, improved analyses of data from run I of the Fermilab Tevatron collider have been providing important complementary constraints [2]. The transition is marked by the termination of the LEP II experimental program in late 2000 and the anticipated start of run II of the Tevatron collider in 2001.

The results of experimental searches for different MSSM particles can usefully be compared and combined using the conventional parametrization of the model in terms of supersymmetry-breaking scalar and gaugino masses $m_{0}, m_{1 / 2}$, the Higgsino mixing parameter $\mu$, the ratio of Higgs vacuum expectation values (VEV's) $\tan \beta$ and a universal trilinear supersymmetry-breaking parameter $A$. We work in the framework of gravity-mediated models of supersymmetry breaking, in which it is commonly assumed that the scalar masses $m_{0}$ and the gaugino masses $m_{1 / 2}$ are universal at some supersymmetric grand unified theory (GUT) scale. The assumptions that these supersymmetry-breaking parameters are universal should be questioned, particularly for scalar masses and especially those of the Higgs supermultiplets, but provide a convenient way of benchmarking comparisons and combinations of different experimental searches. In this paper, we make such comparisons and combinations in variants of the MSSM in which the scalar-mass universality assumption is extended to Higgs fields [univer- sal Higgs boson mass (UHM), also commonly referred to as minimal supergravity (MSUGRA) or the constrained MSSM (CMSSM)], and also without this supplementary assumption [nonuniversal Higgs boson mass (NUHM)].

In making such comparisons, we emphasize the importance of including radiative corrections to the relations between these MSSM model parameters $\left(m_{0}, m_{1 / 2}, \mu, \tan \beta, A\right)$ and the physical masses of MSSM particles. Radiative corrections are well-known to be crucial in the MSSM Higgs sector, but also should not be neglected in the chargino, neutralino, gluino and squark sectors. As we have emphasized previously [3], the differences between the domains of MSSM parameter space apparently explored at the tree and one-loop levels are comparable to the differences between the domains explored in successive years of LEP running at higher center-of-mass energies. In view of the intense experimental effort put into sparticle searches at LEP II, it is important that the final results of these efforts be treated with the theoretical care they deserve. This issue is also relevant if one wishes to compare the physics reaches for electroweakly-interacting sparticles at LEP and for stronglyinteracting sparticles at the Tevatron Collider, in which case one should take into account the important radiative corrections to squark and gluino masses [4], as well as to their production cross sections.

In addition to direct searches for the production of MSSM particles, important indirect constraints must also be taken into account. These include other accelerator constraints, such as the measured value of the $b \rightarrow s \gamma$ decay rate $[5,6]$, and non-accelerator constraints related to the possible role of the lightest supersymmetric particle (LSP) as cold dark matter (CDM). The lightest supersymmetric particle (LSP) would be stable in any variant of the MSSM which conserves $R$ parity, as we assume here. In gravity-mediated 
models of supersymmetry breaking, the framework adopted here, the LSP is commonly thought to be the lightest neutralino $\chi$, and calculations of the cosmological relic density of LSPs, $\Omega_{\chi}$, yield values in the range preferred by cosmology in generic domains of MSSM parameter space [7]. The possibility of supersymmetric CDM provides one of our principal motivations for seeking a deeper understanding of the allowed MSSM parameter space, but is not our only focus in this paper.

The most essential dark-matter constraint is that the relic LSP density not overclose the Universe. The conditions that the universe has an age in excess of 12 billion years and that $\Omega_{\text {total }} \leqslant 1$ imply an upper bound on $\Omega_{\chi} h^{2}$ of 0.3 . Further, the convergent indications from astrophysical structureformation arguments and observations of high-redshift supernovae are that $\Omega_{C D M}<0.5$ [8], whereas the Hubble expansion rate $H_{0}=100 h \mathrm{~km} / \mathrm{s} / \mathrm{Mpc}: h=0.7$ with an error of about $10 \%$ [9], so we require $\Omega_{L S P} h^{2} \leqslant \Omega_{C D M} h^{2} \leqslant 0.3$. On the other hand, astrophysical structure formation seems to require $\Omega_{C D M}>0.2$, so we also require $\Omega_{L S P} h^{2} \geqslant 0.1$, while acknowledging that a lower value of $\Omega_{L S P}$ could be permitted if other CDM particles such as axions and/or superheavy relics are present.

There have recently been some significant developments in the analysis of supersymmetric CDM. One is that the importance of co-annihilation effects involving next-to-lightest supersymmetric particles (NLSPs) such as the $\widetilde{\tau}, \tilde{\mu}$, and $\widetilde{e}$ for calculations of the relic density of a gaugino-like LSP has recently been recognized $[10,11]$. Another phenomenon whose importance in the CMSSM has recently been underlined is the possible transition of the electroweak vacuum into a charge- and color-breaking (CCB) minimum [12]. The absence of such an instability is not absolutely necessary, since a transition in the future cannot be excluded. Therefore, we comment on the regions of MSSM parameter space in which the CCB instability is absent, but do not focus exclusively on these regions.

The main purpose of this paper is to prepare for the compilation, comparison and combination of the definitive results from LEP II and the Tevatron. We illustrate our analysis with the latest available limits from these two experimental programs $[1,2]$, supplemented by educated guesses at their final sensitivities. As we have explained previously, and discuss in more detail below, a key role in constraining the MSSM parameter space is provided by the LEP Higgs search. We express our results as a function of the present LEP lower limit on $m_{H}$, currently $107.9 \mathrm{GeV}$ [13], and the prospective future sensitivity, which may approach $112 \mathrm{GeV}$. We use our analysis to present lower limits on the LSP mass and on $\tan \beta$. We include a discussion of the implications of relaxing the UHM assumption that the soft supersymmetry-breaking contributions to Higgs boson masses are also universal. In particular, we investigate whether a light Higgsino LSP is still a viable dark matter candidate, and find that the latest LEP II data now exclude this possibility. Finally, we discuss the likely future developments in the exploration of the MSSM parameter space in the period before the start-up of the LHC, during which the central role is likely to be played by run II of the Tevatron Collider.

The layout of this paper is as follows. In Sec. II we review in more detail the theoretical framework we adopt, discussing the issues of universality and relic coannihilations, and stressing the importance of Higgs boson mass constraints. In Sec. III we review our implementation of the $b \rightarrow s \gamma$ constraint, including, where applicable, the next-to-leadingorder (NLO) QCD corrections. We discuss the implications of the latest available constraints from LEP II in Sec. IV, combining them in Sec. V with the cosmological and astrophysical constraints $0.1 \leqslant \Omega_{C D M} h^{2} \leqslant 0.3$ as well as the $b$ $\rightarrow s \gamma$ constraint, and making the UHM assumption. We find

$$
m_{\chi} \geqslant 51 \mathrm{GeV}, \quad \tan \beta \geqslant 2.2
$$

and discuss the expanded ranges of $m_{\chi}$ and $\tan \beta$ that may be explored by the improved Higgs-boson mass limits that might be obtained from the run of LEP II in the year 2000. The limits in Eq. (1) are strengthened when we restrict values of $A_{0}$ to minimize the parameter space with CCB minima, in which case we find

$$
m_{\chi} \geqslant 54 \mathrm{GeV}, \quad \tan \beta \geqslant 2.8 .
$$

We further generalize the discussion to nonuniversal Higgs boson masses (NUHM) in Sec. VI, finding that the limits on $m_{\chi}$ and $\tan \beta$ are relaxed to

$$
m_{\chi} \geqslant 46 \mathrm{GeV}, \quad \tan \beta \geqslant 1.9 .
$$

Section VII is devoted to a discussion of the possibility of Higgsino dark matter in such a NUHM scenario. We find that the LEP II searches for charginos, neutralinos and Higgs bosons together now exclude as dark matter an LSP that is more than about $70 \%$ Higgsino. We turn our attention to the Tevatron Collider in Sec. VIII. We compare the LEP II and run I sensitivities to the MSSM parameters, and discuss and compare the regions of MSSM parameter space to which Tevatron run II data should be sensitive [2]. Finally, Sec. IX summarizes our conclusions and the prospects for future improvements and extensions of the analysis reported here.

\section{THEORETICAL FRAMEWORK}

As already mentioned in the Introduction, we work in the context of the MSSM with $R$ parity conserved. We assume a parametrization of soft supersymmetry breaking inspired by supergravity models with gravity mediation from a hidden sector. We assume that the soft supersymmetry-breaking scalar masses $m_{0}$ are universal at the supersymmetric GUT scale, as are the gaugino masses $m_{1 / 2}$ and the trilinear parameters $A$. The renormalization of the physical values of the soft supersymmetry-breaking parameters is then calculated using standard renormalization-group equations. We use two loop renormalization group equations (RGEs) [14] to evolve the dimensionless couplings and the gaugino masses, and one loop RGEs [15] for the other soft masses, and we include one-loop SUSY corrections to $\mu$ [16] and to the top and bottom masses [4] . 

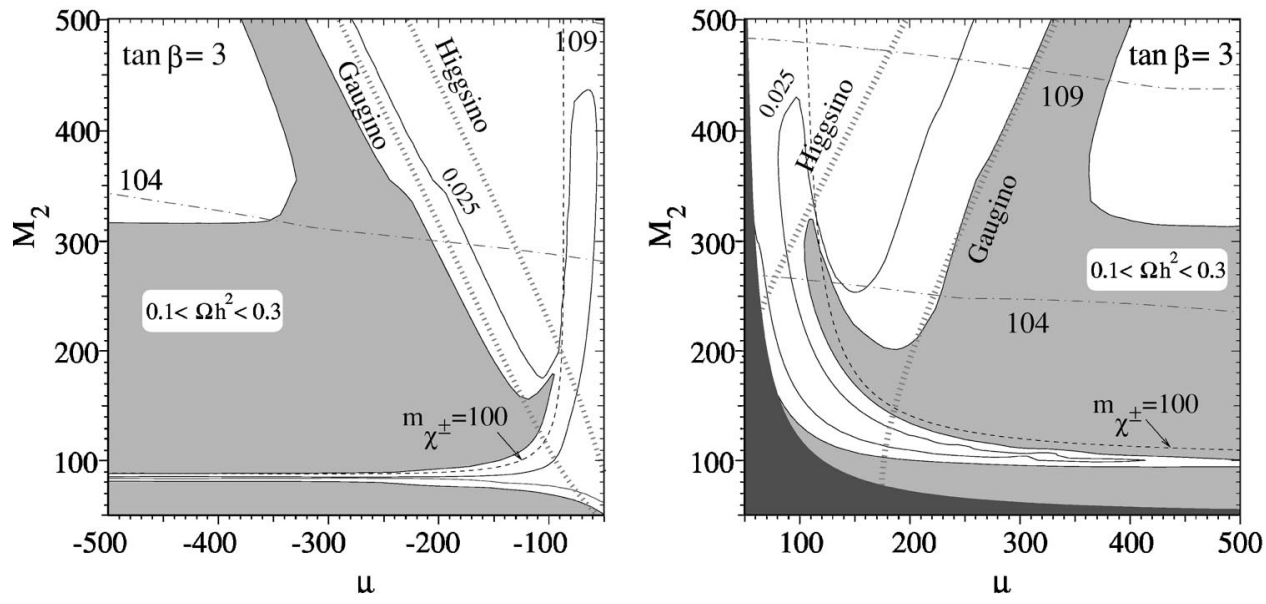

FIG. 1. The $\mu, M_{2}=\left(\alpha_{2} / \alpha_{G U T}\right) \times m_{1 / 2}$ plane for $\tan \beta=3, m_{0}=100 \mathrm{GeV}$ and $m_{A}=1 \mathrm{TeV}$. Contours of $\Omega_{\chi} h^{2}=0.025,0.1$, and 0.3 are shown as solid lines, and the preferred region with $0.1<\Omega_{\chi} h^{2}<0.3$ is shown light-shaded. There are also dashed lines corresponding to $m_{\chi^{ \pm}}=100 \mathrm{GeV}$. The near-horizontal dot-dashed lines are Higgs mass contours, and the hashed lines are 0.9 Higgsino and gaugino purity contours. The dark shaded region has $m_{\chi^{ \pm}}<m_{Z} / 2$.

Deviations from scalar-mass universality could easily be expected, for example in string-motivated models where their magnitudes could be controlled by flavor-dependent modular weights [17]. Upper limits on flavor-changing interactions place restrictions on the possible generationdependences of scalar mass parameters, though these are relatively weak for the third generation. In any case, these do not constrain non-universalities between sparticle fields with different quantum numbers, namely $\tilde{l}_{R}$ vs $\tilde{l}_{L}$ vs $\tilde{q}_{R}$ vs $\tilde{q}_{L}$. Nevertheless, we neglect such possibilities in our analysis. However, although our default option is that universality extends also to the soft supersymmetry-breaking contributions to the Higgs scalar masses (UHM), we do also allow for the possibility that their soft supersymmetry-breaking masses may be non-universal (NUHM).

We use the renormalization-group equations and the oneloop effective potential to implement the constraints of a consistent electroweak vacuum parametrized by the ratio $\tan \beta$ of Higgs VEV's. We therefore adopt a parameterization of the MSSM in which $m_{0}, m_{1 / 2}, A, \tan \beta$ and the sign of $\mu$ are treated as independent parameters, with the magnitude of the Higgsino mixing parameter $\mu$ and the pseudoscalar Higgs boson mass $m_{A}$ (or, equivalently, the bilinear soft supersymmetry-breaking parameter $B$ ) treated as dependent parameters. In the UHM limit, the correlation between $m_{1 / 2}$ and $\mu$ is such that the LSP neutralino $\chi$ typically is mainly a $U(1)$ gaugino $\widetilde{B}$ ( $B$-ino). Since $\mu$ becomes a free parameter (along with $m_{A}$ ) in the NUHM case, $\chi$ may become Higgsino-like for certain parameter choices (roughly $M_{2}$ $>2 \mu)$. Figure 1 gives an overview of the $\mu, M_{2}$ $=\left(\alpha_{2} / \alpha_{G U T}\right) \times m_{1 / 2}$ plane for the illustrative choices $\tan \beta$ $=3, m_{0}=100 \mathrm{GeV}, A_{t}$ at its quasi-fixed point $\sim 2.25 M_{2}$ [18], and $m_{A}=1 \mathrm{TeV}$, showing various contours of the relic density $\Omega_{\chi} h^{2}$, the contour $m_{\chi^{ \pm}}=100 \mathrm{GeV}$, contours of the mass of the lightest MSSM Higgs boson, and contours of Higgsino purity. In this figure we have neglected neutralinoslepton coannihilation (discussed in detail below), since a small change in $m_{0}$ can move the masses out of the coanni- hilation region. We see that the LSP is mainly a $\widetilde{B}$ in most of the $\mu, M_{2}$ plane displayed (where the relic density is of cosmological significance). One of the key questions we investigate is whether a Higgsino LSP is still allowed as a dark matter candidate by LEP II data [3].

The relevance of direct LEP or Tevatron searches for sparticles does not need emphasis. In fact, it turns out that the indirect constraint on the MSSM parameter space provided by the Higgs search is also of great importance, as seen in Fig. 1, particularly in the UHM case. This constraint depends on the MSSM mass parameters, because the mass of the lightest MSSM Higgs boson $h$ is sensitive, via radiative corrections, to sparticle masses, in particular the stop masses. This correlation has some impact even in the NUHM case, as we discuss in more detail later.

It is interesting to confront the range of MSSM parameters still permitted by the LEP and other direct experimental searches for MSSM particles with other less direct experimental constraints, or with theoretical prejudices. Among the latter, one might mention gauge-coupling unification, leptonquark mass unification and the absence of fine tuning. Although we consider all these prejudices appealing, none of them is precise enough to enable us to draw any firm conclusions. Gauge-coupling unification cannot be used to constrain $m_{0}, m_{1 / 2}$, and $\mu$ in the absence of a theory of GUT threshold effects. Lepton-quark mass unification is hostage to uncertainties in neutrino masses and mixing [19]. The fine-tuning price imposed by LEP data is rising [20], particularly for small values of $\tan \beta$, but its interpretation is subjective and no consensus has been reached on the maximal pain that can be tolerated. The constraints we apply in this analysis are rather the indirect experimental ones provided by the measurement of $b \rightarrow s \gamma$ decay [5,6], whose implementation we discuss in the next section, and the cosmological relic-density constraint already mentioned in the Introduction.

In the parameter region of interest, the relic density $\Omega_{\chi} h^{2}$ increases with increasing $m_{0}, m_{1 / 2}$. Therefore the cosmologi- 
cal upper limit $\Omega_{\chi} h^{2} \leqslant 0.3$ may be used here to set upper limits on these soft supersymmetry-breaking parameters. As discussed in the Introduction, strictly speaking there is no astrophysical lower limit on $\Omega_{\chi} h^{2}$, even if one accepts that the cold dark matter $(\mathrm{CDM})$ density $\Omega_{C D M} h^{2} \geqslant 0.1$, since there might be other important sources of CDM, such as axions or ultra-heavy relics. Nevertheless, one may take $\Omega_{\chi} h^{2} \geqslant 0.1$ as a default assumption.

An important recent development has been the recognition that coannihilation of the LSP with next-to-lightest sparticles (NLSPs) may be important [10] in the $B$-ino LSP region that is favored in the UHM case, in particular. Generically, the NLSP in this region is the lighter stau $\tilde{\tau}_{1}$, with the $\tilde{\mu}_{R}$ and $\tilde{e}_{R}$ not much heavier. Since the stable MSSM relic cannot possess electric charge, the allowed region of the MSSM parameter space is bounded by the line $m_{\chi}=m_{\tau_{1}}$. Close to this line, $\chi-\tilde{l}$ and $\tilde{l}-\tilde{l}$ coannihilation effects suppress $\Omega_{\chi} h^{2}$ below the range that would be calculated on the basis of $\chi-\chi$ annihilation alone. This has the effect, in particular, of increasing the maximum allowable value of $m_{1 / 2}$ and hence allowing $m_{\chi} \leqslant 600 \mathrm{GeV}$ for $\Omega_{\chi} h^{2}$ $\leqslant 0.3$. For larger $m_{1 / 2} \geq 400 \mathrm{GeV}$, the allowed range of $m_{0}$ has a typical thickness $\delta m_{0} \sim 30 \mathrm{GeV}$. On the other hand, when $m_{1 / 2} \lesssim 400 \mathrm{GeV}$, there is a relatively broad allowed range for $m_{0}$ between about 50 and $150 \mathrm{GeV}$, depending on $\tan \beta, A$ and the sign of $\mu$.

We have shown previously $[21,3]$ that the lower limit on $m_{\chi}$ imposed by data from LEP and elsewhere may be strengthened by combining it with additional theoretical constraints such as the cosmological relic density. The previous analysis included coannihilation effects only in the Higgsino region. The inclusion of LSP-NLSP coannihilation in the $B$-ino region is less important for the inferred lower limit on $m_{\chi}$, as we discuss later. However, it is important when one is assessing how much of the preferred range of MSSM parameter space may escape searches at LEP and elsewhere.

In our analysis below, we consider parameter ranges that highlight the current experimental bounds and are consistent with the relic cosmological density. We use four default values for $\tan \beta$, namely $3,5,10$, and 20 . Lower values of $\tan \beta$ are disfavored by the LEP Higgs boson mass limit, and the study of higher values would require an improved treatment of the cosmological relic density calculation for large $\tan \beta$, which lies beyond the scope of this paper. Although most of our figures display more restricted ranges of $m_{1 / 2}$, we note that in the UHM case cosmology allows values of $m_{1 / 2}$ up to $\sim 1400 \mathrm{GeV}$. We consider two possible treatments of the trilinear soft supersymmetry-breaking parameter $A_{0}$ at the GUT scale which we assume to be universal. The conservative approach in the UHM case is to vary $A_{0}$ so as to minimize the impact of the accelerator constraints $\left(\mathrm{UHM}_{\min }\right)$, and the other is to choose [12] $A_{0}=-m_{1 / 2}$ so as to maximize the area in the $m_{0}-m_{1 / 2}$ parameter plane in the present electroweak vacuum is stable, and CCB minima are irrelevant. In the NUHM case, we must also specify values of $\mu$ and $m_{A}$. For the purpose of translating Higgs boson mass limits into limits in the $m_{0}-m_{1 / 2}$ plane, we fix $m_{A}=10 \mathrm{TeV}$, so as to maximize the light Higgs scalar mass and therefore derive

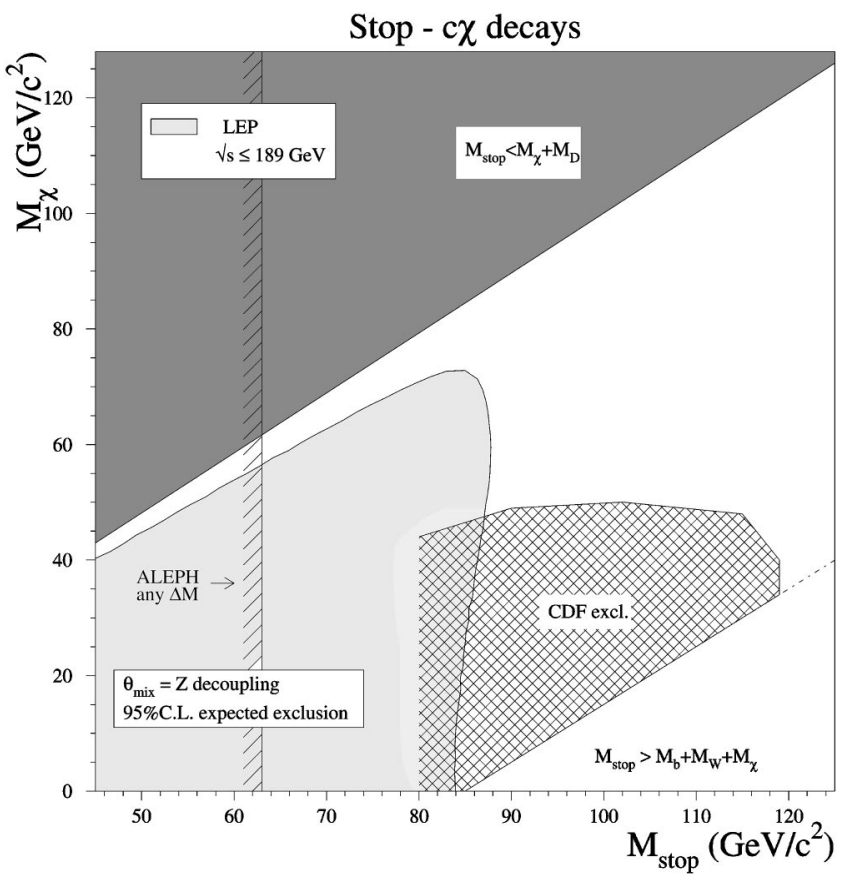

FIG. 2. Present constraints in the $\left(m_{\tilde{t}}, m_{\chi}\right)$ plane assuming a $100 \%$ branching ratio for the decay process $\tilde{t}_{1} \rightarrow u / c \chi$. The vertical hatched band represents the recent ALEPH exclusion valid for any $\Delta M$ value presented in [22]; the light gray region is the LEPcombined excluded region using data up to $\sqrt{s}=189 \mathrm{GeV}$ under the most conservative assumption for the coupling $Z \tilde{t}_{1} \overline{\tilde{t}}_{1}$ [23]; the cross-hatched area is the CDF exclusion using the complete run I data sample [24].

the most conservative bound possible. We allow $\mu$ and $A_{0}$ to vary as much as possible while remaining consistent with the experimental lower bounds on the sparticle masses.

There are restrictions on large values of $A_{0}$, so as to ensure that the sfermion masses are well-behaved. One of the most stringent bounds is that imposed by the experimental lower limit on the lighter stop mass, which depends on $m_{\chi}$ in the way depicted in Fig. 2, which combines the constraints from [22-24]. Another important requirement is that the LSP not be a stau: $m_{\tau_{1}}>m_{\chi}$. The impacts of the stop and stau constraints are illustrated in Fig. 3. We show for $\mu>0$ and $\tan \beta=3,5,10$, and 20 the corresponding upper limits on $A_{0}$ in the UHM case as functions of $m_{1 / 2}$ for $m_{0}=100 \mathrm{GeV}$. Also shown in Fig. 3 as broken lines are representative Higgs boson mass contours. It is apparent that the Higgs boson mass is very sensitive to the value of $A_{0}$. The corresponding figures for $\mu<0$ are similar but allow somewhat higher values for $A_{0}$. The sensitivity of the Higgs boson mass to $A_{0}$ translates into a corresponding sensitivity in the lower limit on $m_{\chi}$.

\section{CONSTRAINTS FROM $b \rightarrow s \gamma$ DECAY}

The width for the inclusive decay $\mathrm{B} \rightarrow \mathrm{X}_{\mathrm{s}} \gamma$ is determined by flavor-violating loop diagrams, and is therefore sensitive to physics beyond the standard model. In generic models with two Higgs doublets, significant contributions come 

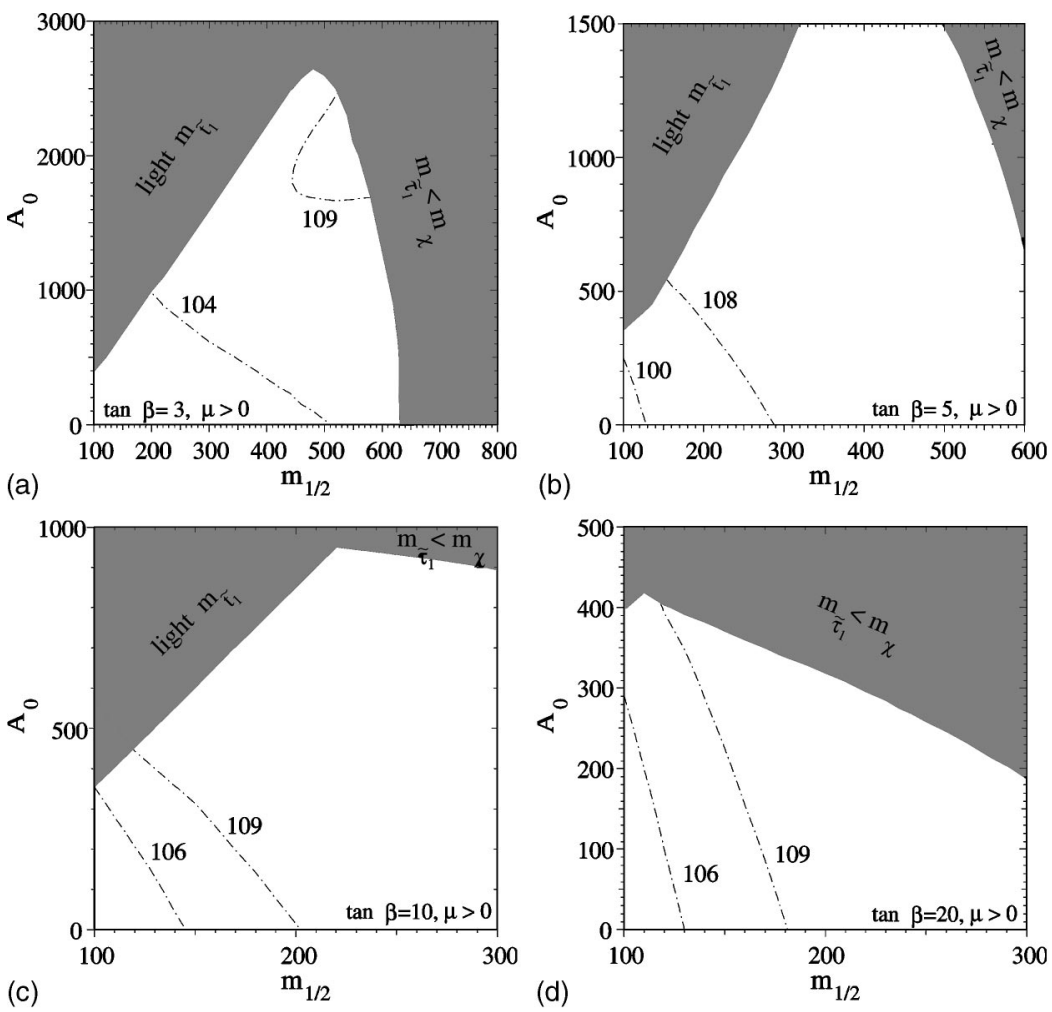

FIG. 3. Upper bounds on the trilinear supersymmetry-breaking parameter $A_{0}$, for $\tan \beta=3,5,10$, and 20 and $\mu>0$, as a function of $m_{1 / 2}$. The shaded regions yield either a tachyonic $\tilde{t}$ or a $\tilde{\tau}$ LSP. Also shown is the dependence of the lightest Higgs boson mass on $m_{1 / 2}$ and $A_{0}$.

from charged Higgs boson exchange, which always increases the $\mathrm{SM}$ prediction for $\mathcal{B}_{s \gamma} \equiv B\left(\mathrm{~B} \rightarrow \mathrm{X}_{\mathrm{s}} \gamma\right)$, allowing severe lower limits on the mass of the charged Higgs boson to be set: see [25], for instance. However, these limits do not apply directly to supersymmetric extensions of the standard model, because, in addition to the two Higgs doublets, there are chargino-stop contributions which can interfere destructively with the charged Higgs boson ones, and thereby reduce the predicted rate for $\mathcal{B}_{s \gamma}[26]$.

Calculations including next-to-leading order (NLO) QCD corrections exist for both the standard model and generic two-Higgs-doublet models (see [25] and references therein), whereas in the case of supersymmetry the leading order (LO) calculations [27] have been complemented with NLO QCD corrections that are valid only under certain assumptions [28].

We include in our numerical analysis a $\mathcal{B}_{s \gamma}$ calculation based on the full NLO treatment for the standard model and charged Higgs contributions, and the best available ${ }^{1}$ treatment of QCD corrections to the supersymmetric contributions [28]. The latter turned out to be of limited applicability in our analysis, since the conditions in which they well approximate the whole NLO supersymmetric corrections are usually not met. Therefore, the results presented below are

\footnotetext{
${ }^{1}$ The code implementing these calculations has been kindly provided to us by P. Gambino, who also helped in designing a recipe to determine the applicability of the supersymmetric NLO calculations.
}

based mostly on the LO supersymmetric contributions only. The prediction for $\mathcal{B}_{s \gamma}$ depends on some experimental inputs and on three renormalization scales. The experimental inputs are the top-quark mass, the Cabibbo-Kobayashi-Maskawa (CKM) mixing-angle factor $\left|V_{t b} V_{t s}^{*} / V_{c b}\right|$, the $c$ and $b$ quark masses, the inclusive semileptonic branching fraction of $B$ hadrons $B_{\text {lept }, X}$, the strong coupling $\alpha_{s}\left(M_{Z}\right)$, and the electromagnetic coupling $\alpha_{e m}$. The renormalization scales are those relevant to the semileptonic and radiative processes $\sim m_{b}$, and the high-energy matching scale $\sim M_{W}$. We used as nominal values and errors for these quantities those quoted in Table 1 of [25], except for $B_{\text {lept }, X}, \alpha_{s}\left(M_{Z}\right)$ and $\alpha_{e m}$. For the former we used the latest average provided by the LEP electroweak working group [29], $B_{\text {lept }, X}=0.1058$ \pm 0.0018 . For $\alpha_{s}\left(M_{Z}\right)$ we took the latest Particle Data Group (PDG) [30] combination: 0.119 \pm 0.002 . Finally, for $\alpha_{e m}$ we took the value at $q^{2}=0$ following [28]. For each given point in the parameter space, we determined the theoretical error $\delta \mathcal{B}_{s \gamma}^{\text {theor }}$ as the RMS of $1000 \mathcal{B}_{s \gamma}$ values obtained by varying the experimental inputs with independent and Gaussian errors. Moreover, we determined the reference theoretical prediction for $\mathcal{B}_{s \gamma}^{\text {theor }}$ conservatively, as the value closest to the measured one that we could obtain by varying independently the three renormalization scales from half to twice their nominal value.

The experimental measurements of the rate for the inclusive process $\mathrm{B} \rightarrow \mathrm{X}_{\mathrm{s}} \gamma[5,6]$ are dominated by the latest CLEO result

$$
\mathcal{B}_{\text {sy }}^{\text {meas }}=(3.15 \pm 0.35 \pm 0.32 \pm 0.26) \times 10^{-4} \text {, }
$$



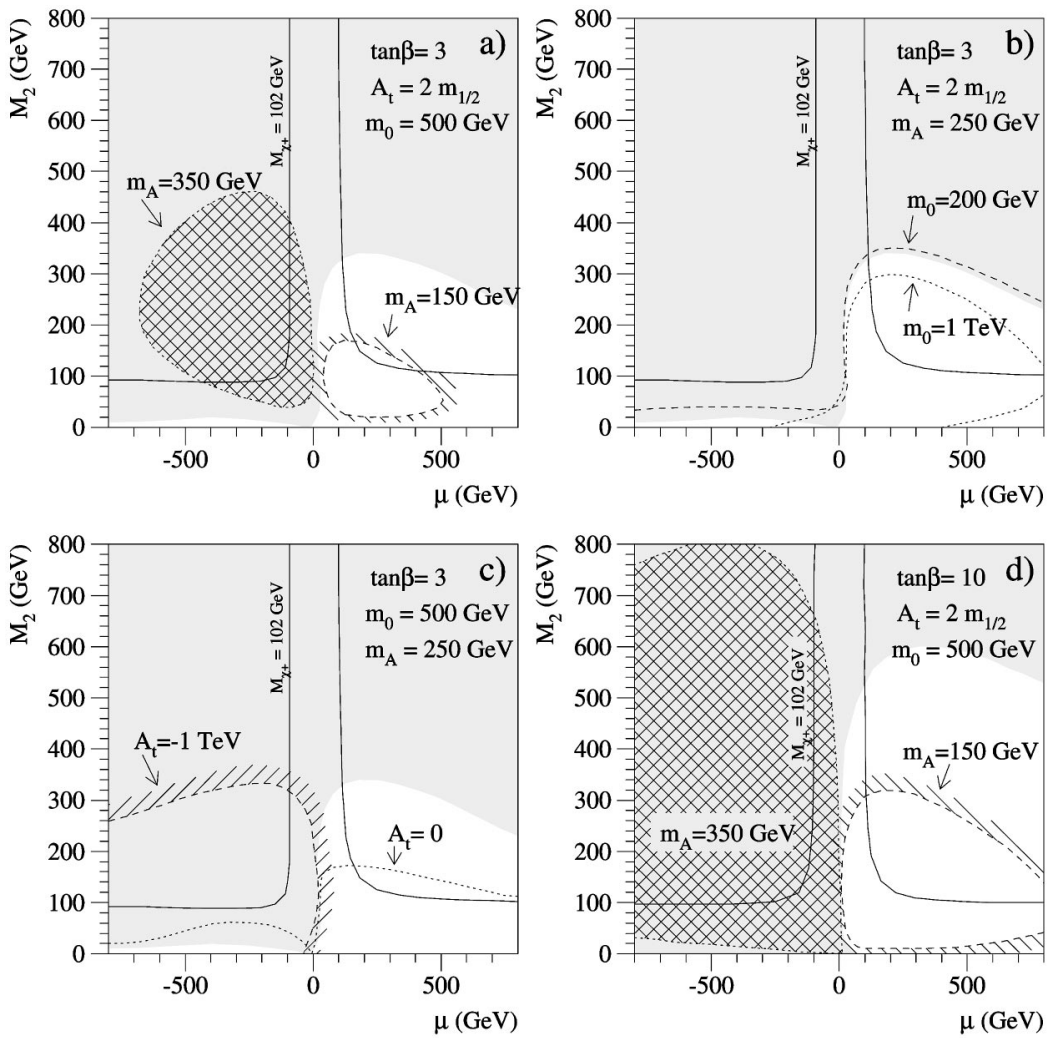

FIG. 4. Constraints on the NUHM parameter space imposed by $\mathcal{B}_{s \gamma}$ : domains in the $\left(\mu, M_{2}\right)$ plane excluded for tan $\beta=3$ (a,b,c) and $\tan \beta=10$ (d). In all plots the "reference" excluded region for $m_{A}=250 \mathrm{GeV}, m_{0}=500 \mathrm{GeV}$ and the infra-red quasi-fixed-point value $A_{0}=2 m_{1 / 2}$ is shaded, assuming $m_{t}=175 \mathrm{GeV}$. The effect of varying $m_{A}$ is shown in panel (a), the effect of varying $m_{0}$ is shown in panel (b), the effect of changing the sign of $A$ is shown in panel (c), and panel (d) illustrates the effect of increasing tan $\beta$. Please see the text for further details.

which is in good agreement with the SM prediction of $(3.29 \pm 0.33) \times 10^{-4}[31]$. There is therefore no need for any physics beyond the standard model, and we establish only upper limits on the possible supersymmetric contributions.

To determine the $95 \%$ confidence-level exclusion domain in the supersymmetric parameter space, we treated separately the contributions to the CLEO error. The three terms in Eq. (3) come from limited statistics, experimental systematics and model dependence, respectively. By adding in quadrature the first two terms we defined $\delta \mathcal{B}_{s \gamma}^{\text {meas }}$ which we treated as a Gaussian error, whilst we considered the third one, $\delta \mathcal{B}_{s \gamma}^{\text {model }}=0.26$, as an additional scale error. We have then defined a $\chi^{2}$ function

$$
\chi^{2} \equiv \frac{\left(\left|\mathcal{B}_{s \gamma}^{\text {meas }}-\mathcal{B}_{s \gamma}^{\text {theor }}\right|-\delta \mathcal{B}_{s \gamma}^{\text {model }}\right)^{2}}{\left(\delta \mathcal{B}_{s \gamma}^{\text {meas }}\right)^{2}+\left(\delta \mathcal{B}_{s \gamma}^{\text {theor }}\right)^{2}},
$$

and regard as excluded those points giving a $\chi^{2}$ probability for one degree of freedom smaller than $5 \%$.

We have investigated the impact of the $\mathcal{B}_{s \gamma}$ constraint (4), (5) in the $\left(\mu, M_{2}\right)$ plane, without making the UHM assumption. Figure $4 \mathrm{a}$ shows the domains excluded by $\mathcal{B}_{s \gamma}$ as a function of $m_{A}$ for $\tan \beta=3, m_{0}=500 \mathrm{GeV}$, and $A_{t}$
$=2 m_{1 / 2}$, the quasi-fixed point value. ${ }^{2}$ The kinematic reach for charginos at $\sqrt{s}=204 \mathrm{GeV}$ is also shown for comparison. As expected, the extent of the excluded region depends strongly on $m_{A} \simeq \sqrt{m_{H^{ \pm}}^{2}-m_{W}^{2}}$, essentially vanishing when $m_{A}>350-400 \mathrm{GeV}$. When $m_{A}=350 \mathrm{GeV}$, the excluded region collapses to the cross-hatched area shown in Figs. 4a and $4 \mathrm{~d}$. Focusing on the case of $m_{A}=250 \mathrm{GeV}$, at large $M_{2}$ either the chargino or the top squark is heavy enough to suppress the supersymmetric contributions to $\mathcal{B}_{s \gamma}$. In this case, the positive charged-Higgs-boson contribution dominates, making the predicted value of $\mathcal{B}_{s \gamma}$ incompatible with the measured value. For moderate $M_{2}$ values, the supersymmetric contribution becomes sizeable. When $\mu>0$, it interferes negatively with the charged Higgs contribution, reducing the predicted value for $\mathcal{B}_{s \gamma}$, whereas for $\mu<0$ it adds constructively to the charged Higgs contribution, strengthening the exclusion. This explains the shape of the excluded domains for $m_{A}=150,250 \mathrm{GeV}$. The excluded domains depend only mildly on $m_{0}$, as shown in Fig. 4b, whilst it can be seen in Fig. $4 \mathrm{c}$ that the dependence on the sign of $A_{t}$ is significant, and we also show for comparison the case $A_{t}$

\footnotetext{
${ }^{2}$ Note that, in this figure alone, we display plots for the renormalized low-energy value $A_{t}$, rather than the input GUT value $A_{0}$.
} 
$=0$. Finally, Fig. $4 \mathrm{~d}$ shows the same exclusion domains as Fig. $4 \mathrm{a}$, but for for $\tan \beta=10$. While the charged Higgs contributions essentially saturate for $\tan \beta>4-5$, the supersymmetric contributions contain terms of order $1 / \cos \beta$, and therefore increase with $\tan \beta$. When $\mu>0$, this has the effect of further reducing the prediction and hence the excluded region, while, for $\mu<0$, it enhances $\mathcal{B}_{s \gamma}$, thereby extending the sensitivity of these constraints to larger $m_{A}$ values.

\section{UPDATE ON CONSTRAINTS FROM LEP II}

The latest general presentations of results from the four LEP Collaborations were made in 2000 [32]. They were based on the following mean integrated luminosities in each experiment at the indicated center-of-mass energies:

$$
\begin{aligned}
& E_{C M}=188.6 \mathrm{GeV}: 171 \mathrm{pb}^{-1} \\
& E_{C M}=191.6 \mathrm{GeV}: 28 \mathrm{pb}^{-1} \\
& E_{C M}=195.6 \mathrm{GeV}: 78 \mathrm{pb}^{-1} \\
& E_{C M}=199.6 \mathrm{GeV}: 80 \mathrm{pb}^{-1} \\
& E_{C M}=201.6 \mathrm{GeV}: 38 \mathrm{pb}^{-1} .
\end{aligned}
$$

No significant signals were announced in any sparticle or Higgs search channel. Numerical lower limits on the Higgs boson masses were presented separately by the four experiments, and a preliminary combination performed by the LEP Higgs Working Group is available [13]. Limits were also presented by the individual experiments on sparticle production within several frameworks, but the combination of the standard channels usually provided by the LEP Supersymmetry Working Group [1] was not made available at this time. We extrapolate the available combined LEP limits, provided on the basis of the running up to $E_{C M}$ $=188.6 \mathrm{GeV}$ for the sparticles and up to $E_{C M}$ $=201.6 \mathrm{GeV}$ for the Higgs bosons, to include the higherenergy/luminosity data.

We consider the following possible scenarios for the future evolution of the integrated LEP luminosity. The pessimistic one is that no significant additional high-energy luminosity is accumulated (remember the beer bottles?). In this case, the sparticle and Higgs sensitivity will remain essentially as they are at the end of 1999 . We believe that a more realistic scenario is for LEP to accumulate luminosity at the same average rate of $1.3 \mathrm{pb}^{-1}$ as in 1999 , but at somewhat higher energies, say $2 / 3$ at $E_{C M}=202 \mathrm{GeV}$ and $1 / 3$ at $E_{C M}$ $=204 \mathrm{GeV}$. This would result in the following total integrated luminosities per experiment at energies above $E_{C M}$ $=200 \mathrm{GeV}$ :

$$
\begin{aligned}
& E_{C M}=202.0 \mathrm{GeV}: 160 \mathrm{pb}^{-1} \\
& E_{C M}=204.0 \mathrm{GeV}: 60 \mathrm{pb}^{-1} .
\end{aligned}
$$

A more optimistic scenario would be that luminosity is accumulated at a rate sometimes achieved in 1999, but not consistently, and that $50 \%$ of the running is at $E_{C M}$ $=204 \mathrm{GeV}$ and $206 \mathrm{GeV}$ :

$$
\begin{aligned}
& E_{C M}=202.0 \mathrm{GeV}: 140 \mathrm{pb}^{-1} \\
& E_{C M}=204.0 \mathrm{GeV}: 80 \mathrm{pb}^{-1} \\
& E_{C M}=206.0 \mathrm{GeV}: 20 \mathrm{pb}^{-1} .
\end{aligned}
$$

We do not provide detailed results for this optimistic scenario, but do make some comments on its potential impact.

The sparticle final states of relevance for this analysis are $\chi^{+} \chi^{-}, \chi \chi^{\prime}, \chi^{\prime} \chi^{\prime}$, and $\tilde{l}^{+} \tilde{l}^{-}$. In addition, the experimental limits on the squark-production processes $\tilde{t} \bar{t}$ and $\tilde{b} \bar{b}$ can be used to infer constraints on the $A$ parameters, as discussed in Sec. III. We contrast two approaches in the following, either we take the CCB constraint into account and fix $A_{0}$ so as to minimize its impact, or we take a conservative approach, allowing any value of $A$ consistent with the experimental limits on $m_{\tilde{t}_{1}}$ and other constraints $\left(\mathrm{UHM}_{\min }\right)$.

The experimental efficiency for sparticle detection and hence the cross-section upper limit depends on other parameters besides the target sparticle mass, for example the mass difference $\Delta M$ in the sparticle decay, e.g., $\chi^{+} \rightarrow \chi+X$. We have modeled these varying detection efficiencies using a multistep function, with a lower $\Delta M$ cutoff, low and high $\Delta M$ regions. We have used the available publications by the LEP Collaborations and the documentation provided by the LEP Supersymmetry Working Group [1] to derive reasonable values for the transitional values of $\Delta M$ and the average efficiency values within the two regions. We did the same for the background contaminations, except in the case of slepton production, in which case we modeled the dominant $W^{+} W^{-}$ background in different regions of the $\left(M_{\tilde{l}}, M_{\chi}\right)$ plane using its detailed kinematics. We have checked that our parametrization reproduces the available published results. In each of the "realistic" and "optimistic" scenarios $(8,9)$, we make the assumption that the experimental efficiencies and contaminations remain similar to those at $E_{C M} \leqslant 189 \mathrm{GeV}$, based on the fact that the properties of the standard process do not change dramatically in the spanned energy range. Examples of the estimated upper limits on sparticle production cross sections that we obtain from our extrapolation to the three LEP running scenarios discussed above are given in Table I.

For charginos, we conservatively assume no detection efficiency for $\Delta M<5 \mathrm{GeV}$. For larger $\Delta M$ values, the estimated upper limits allow one to exclude chargino production up to a few hundred $\mathrm{MeV}$ below the kinematic limit, unless sneutrino masses, and hence $m_{0}$, are very small. In the case of associated neutralino production, we combine all the kinematically accessible channels, weighted by their visible cross sections, i.e., we take into account the branching fractions into $\chi \nu$ final states, and the estimated efficiencies. We found that the estimated upper limits depend only weakly on the point in the MSSM parameter space, and typical values are given in Table I. In the case of slepton production, we set 
TABLE I. Examples of estimated upper limits on sparticle cross sections (in pb): for charginos and sleptons, assuming $M_{\chi}$ $=50 \mathrm{GeV}, M_{\chi^{ \pm}}=100, M_{\tilde{e}^{ \pm}}=95$, and $M_{\tilde{\tau}^{ \pm}}=90 \mathrm{GeV}$, respectively. In the case of neutralino production, typical values are given.

\begin{tabular}{cccc}
\hline \hline LEP scenario & 1999 & "realistic" $2 \mathrm{~K}$ & "optimistic" $2 \mathrm{~K}$ \\
$\operatorname{Max} \sqrt{s}(\mathrm{GeV})$ & 201.6 & 204 & 206 \\
\hline$\chi^{+} \chi^{-}$ & 0.19 & 0.11 & 0.11 \\
$\tilde{e}^{+} \tilde{e}^{-}$ & 0.06 & 0.05 & 0.06 \\
$\tilde{\tau}^{+} \tilde{\tau}^{-}$ & 0.09 & 0.08 & 0.08 \\
$\chi_{i}^{0} \chi_{j}^{0}$ & $\sim 0.08$ & $\sim 0.08$ & $\sim 0.07$ \\
\hline \hline
\end{tabular}

the $\Delta M$ cutoff at $3 \mathrm{GeV}$. As an example, Fig. 5 shows the exclusions we obtain in the plane $\left(m_{\tilde{e}_{R}}, m_{\chi}\right)$ for $\tan \beta=3, \mu$ $=200 \mathrm{GeV}$ and $\operatorname{BR}\left(\tilde{e}_{R}^{ \pm} \rightarrow \chi e^{ \pm}=100 \%\right)$, under the different hypotheses for LEP running in 2000.

We now turn to the estimation of upper limits on Higgs boson production. In order to estimate the prospects for standard model Higgs limits from the reaction $e^{+} e^{-} \rightarrow h Z^{0}$, we take the simple parametrization of the LEP limits obtained from data up to $E_{C M} \sim 189$ discussed in [33]. Extrapolating this parametrization to include the 1999 data set (6) leads to the estimated limit $m_{H} \geqslant 109 \mathrm{GeV}$. This result is in good agreement with the expected limit reported in [13]. However, the observed limit quoted in the same reference is 107.9 $\mathrm{GeV}$ : the difference is explained as a statistical fluctuation in the data at the level of one standard deviation.

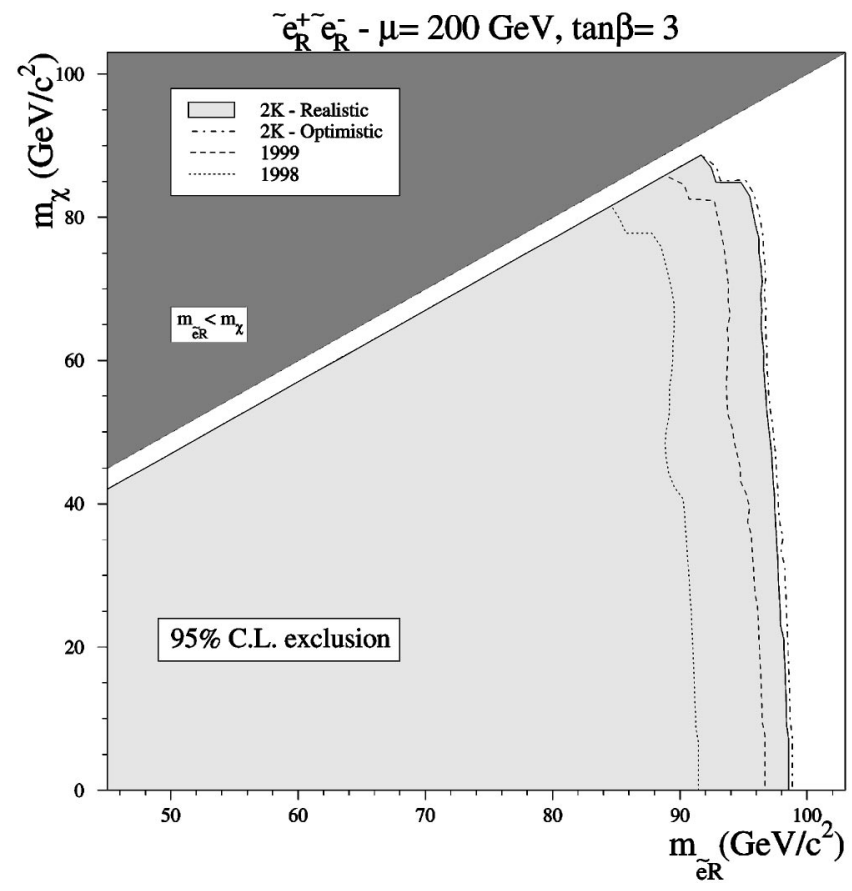

FIG. 5. Constraints in the $m_{\tilde{e}_{R}}, m_{\chi}$ plane imposed by the combined LEP data at $E_{C M} \leqslant 189 \mathrm{GeV}$ (dotted line), our estimates for the limits obtainable by combining the LEP data taken in 1999 (7) (dashed line), and our estimates for the possible 2000 exclusions in the "realistic"' (8) (solid line) and "optimistic" (8) (dot-dashed line) scenarios described in the text.

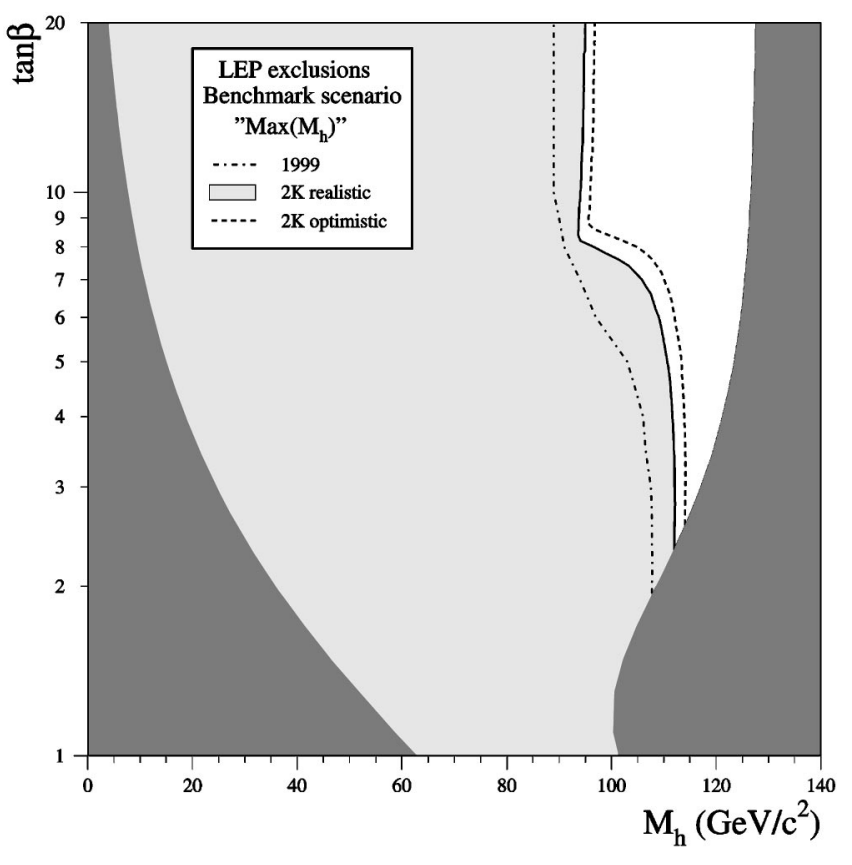

FIG. 6. Constraints in the $m_{h}, \tan \beta$ plane imposed in the "Max $\left(M_{h}\right)$ "' benchmark scenario by combining the LEP data taken in 1999 (7) (dot-dashed line), and our estimates for the possible 2000 exclusions in the "realistic" (8) (solid line) and "optimistic" (9) (dashed line) scenarios described in the text. Also shown (darkshaded) are the regions of the $m_{h}, \tan \beta$ plane excluded by theoretical calculations [34].

The corresponding limits for the "realistic" and "optimistic" running scenarios for the year 2000 are 112 and 114 $\mathrm{GeV}$, respectively, following roughly the empirical rule $M_{H} \geqslant E_{C M}-93 \mathrm{GeV}$. Similar estimates apply to the MSSM for small $\tan \beta \lesssim 5$, as shown in Fig. 6 . $^{3}$ For larger values of $\tan \beta$, we use the same limiting cross section for $e^{+} e^{-}$ $\rightarrow h Z^{0}$, which gives a weaker lower limit on $m_{h}$, because of the smaller $Z^{0} Z^{0} h$ coupling. When $\tan \beta \geq 8$, the production mechanism $e^{+} e^{-} \rightarrow h A$ becomes important, which we include in our analysis following again the prescription given in [33]. Our estimated limiting curves in the $m_{h}, \tan \beta$ plane shown in Fig. 6 have been calculated in the "Max $\left(M_{h}\right)$ ", benchmark scenario suggested in [34]. We have not attempted to combine the $h Z^{0}$ and $h A$ analyses, but have only overlapped them, so our results could be considered conservative in the intermediate-tan $\beta$ region. We indicate in Fig. 6 the LEP limit for the full 1999 data set (dot-dashed line), our estimate for the "realistic" 2000 running scenario (8) (shaded) and the "optimistic" scenario (9) (dashed line). Also shown (shaded) are the regions of the $m_{h}, \tan \beta$ plane excluded by theoretical calculations [34].

Some caution is required when we compare our results directly with the lower limits given by the LEP experiments because of theoretical uncertainties in the MSSM Higgs boson mass calculations. Conservatively, we allow for an error

\footnotetext{
${ }^{3}$ We have verified that these limits are not weakened by the appearance of invisible decay modes $h, A \rightarrow \chi \chi$.
} 

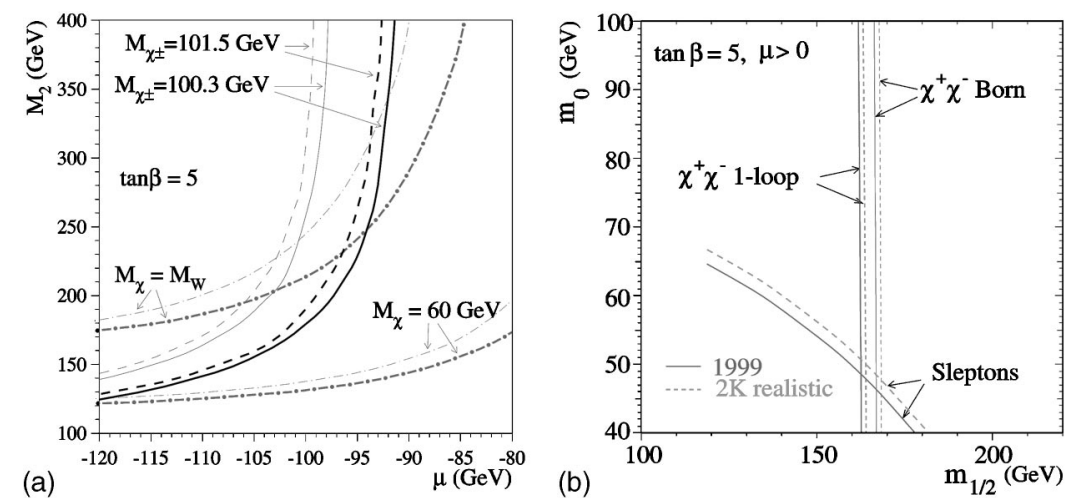

FIG. 7. The effects of radiative corrections to chargino masses in (a) the $\left(\mu, M_{2}\right)$ plane for $\tan \beta=5$ and (b) the $\left(m_{1 / 2}, m_{0}\right)$ plane for $\tan \beta=5$ and $\mu>0$. The contours obtainable from the 1999 and "realistic" $2 \mathrm{~K}$ data are indicated, both with (thicker lines) and without radiative corrections (thinner lines). We notice that the differences between the lines with and without radiative corrections are larger than those between the 1999 and $2 \mathrm{~K}$ lines.

of $\sim 3 \mathrm{GeV}$ in these, so that we translate the experimental limits into the supersymmetric parameter space using the theoretical contours for $104 \mathrm{GeV}$ (for $\tan \beta=3$ ) and 100 $\mathrm{GeV}$ (for $\tan \beta=5$ ) in the case of the complete 1999 data LEP scenario, and $109 \mathrm{GeV}$ and $108 \mathrm{GeV}$, respectively for the two values of $\tan \beta$, in the "realistic'" 2000 LEP scenario. At the higher values of $\tan \beta$ considered, the Higgs boson mass limits do not provide strong constraints and are not used.

We stress that radiative corrections to chargino and neutralino masses, though less dramatic than to Higgs boson masses, are also relevant to the interpretation of experimental limits on physical particle masses in terms of constraints on MSSM parameters such as $\mu, m_{1 / 2}$ and $m_{0}$ [3]. Two such effects are seen in Fig. 7. We see that the differences between the 1999 and $2 \mathrm{~K}$ limits are considerably smaller than the shifts induced by the radiative corrections. In particular, as shown in the $\mu, m_{1 / 2}$ plane in panel (a), radiative corrections are very significant in the delicate Higgsino region discussed in Sec. VII. Their inclusion is indispensable for the accurate interpretation of the LEP data, as we do throughout this paper.

\section{THE CASE OF UNIVERSAL HIGGS BOSON MASSES}

We next apply the above accelerator constraints under the assumption that the soft supersymmetry breaking masses are universal, including the Higgs multiplets (UHM), exploring their impact in the the $m_{1 / 2}, m_{0}$ parameter plane and comparing them with the constraints from cosmology on the relic abundance of the LSP. We remind the reader that, in this UHM context, for fixed $\tan \beta$ and sign of $\mu$, the only parameter choice remaining is the value of $A_{0}$. We discuss below two cases, one in which we require the absence of charge and color breaking (CCB) minima [12], but fix $A_{0}=-m_{1 / 2}$ so as to minimize their impact, and the other in which we disregard CCB minima, and allow $A_{0}$ to vary freely $\left(\mathrm{UHM}_{\min }\right)$.

We start with the CCB UHM case shown in Figs. 8 and 9. One is safe from CCB minima above the curved solid lines in these plots, which are calculated with $A_{0}=-m_{1 / 2}$, so as to minimize the impact of this prospective constraint. Plotted as near-vertical dashed lines are the chargino mass contours: $m_{\chi}^{ \pm}=102$. At the higher values of $\tan \beta$, the bound on $m_{1 / 2}$ from the chargino mass limit is nearly independent of $\tan \beta$, and the dependence is always very slight for $\mu>0$. For completeness we also show the limit from the selectron mass bound.

The light shaded regions in Fig. 8 are those excluded by the $b \rightarrow s \gamma$ constraint discussed in Sec. III. We see that, for $\mu<0$, the impact of $\mathcal{B}_{s \gamma}$ constraints increases sizeably with $\tan \beta$, and covers in these cases a significant fraction of the region otherwise preferred for dark matter reasons. The cutoff at large $m_{0}, m_{1 / 2}$ is due to the corresponding increase of $m_{A}$, and hence $m_{H^{ \pm}}$, in the UHM, which reduces the charged Higgs contribution. The exclusion is not very sensitive to the $A_{0}$ value chosen. As we can see in Fig. 9, for $\mu$ $>0$ the interference between the supersymmetric and charged Higgs contributions cancel the effect of new physics in the low-medium $\tan \beta$ range; however, there is still some sensitivity at large $\tan \beta$ where the large negative supersymmetric contribution make $\mathcal{B}_{s \gamma}$ significantly smaller than the measured value, as seen in panel (d) of Figs. 9.

Also shown in these figures by near-vertical dot-dashed lines are the limits coming from the Higgs boson mass bounds. Note that for $\mu<0$, these contours only appear for $\tan \beta=5$, where we display the 100 and $108 \mathrm{GeV}$ contours corresponding to the 1999 and prospective "realistic", 2K experimental limit, allowing a safety margin of $3 \mathrm{GeV}$ as discussed earlier. At $\tan \beta=3$, the position of these contours is far off to the right, excluding the entire region displayed. At $\tan \beta=10$ and 20 , the contours would appear to the left of the chargino bound and are not shown. For $\mu>0$, the limits are weaker, i.e., the contours move to the left. In the case of $\tan \beta=3$, the $102 \mathrm{GeV}$ and $104 \mathrm{GeV}$ contours have now moved into the displayed range of $m_{1 / 2}$, and the contours for $100 \mathrm{GeV}$ and $108 \mathrm{GeV}$ are shown for $\tan \beta=5$. In Fig. 10, we show an extended range in $m_{1 / 2}$ and the position of the $104 \mathrm{GeV}$ contour for $\tan \beta=3, \mu>0$.

The Higgs boson mass contours depend on $m_{0}$, because the radiative corrections to the Higgs boson mass cause these curves to bend left at very large $m_{0}$. Thus ultimately, the 

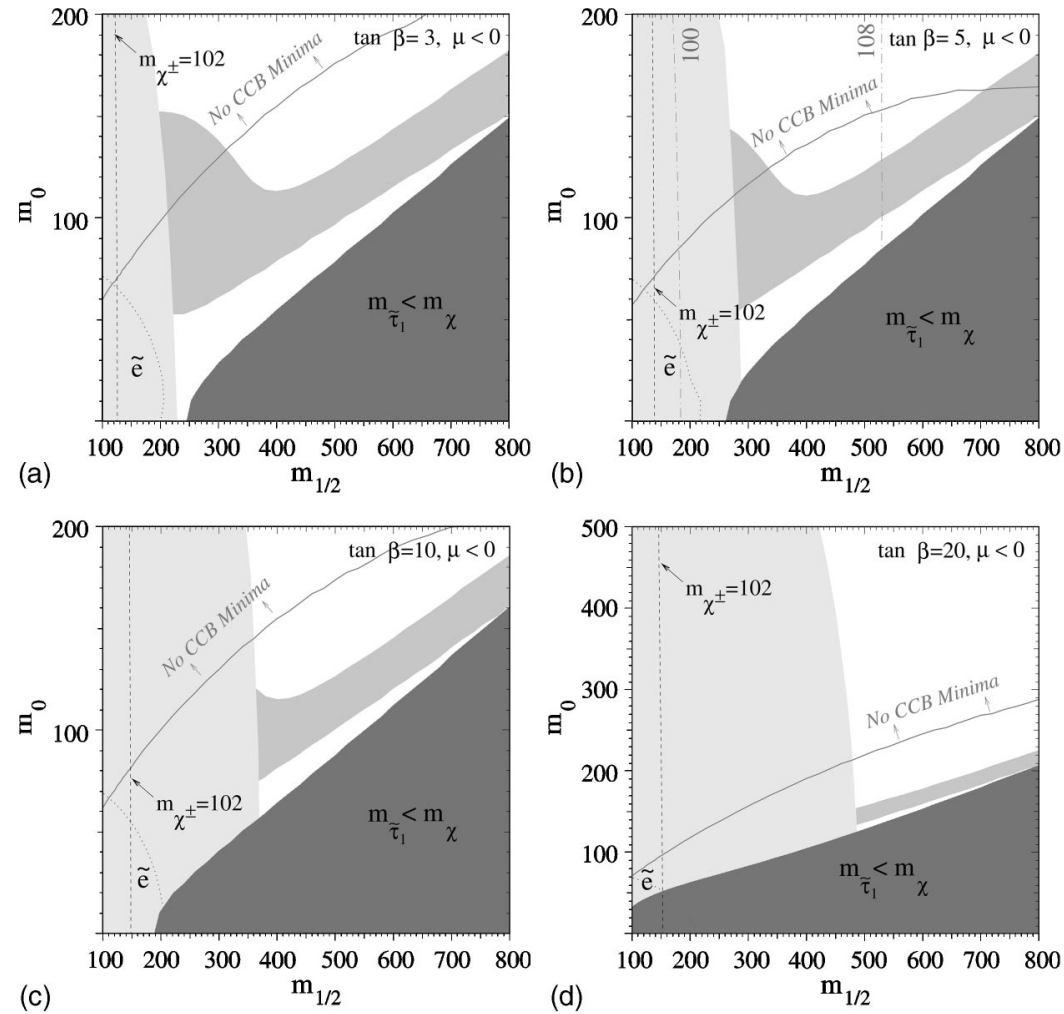

FIG. 8. The $m_{1 / 2}, m_{0}$ plane for $\mu<0, A_{0}=-m_{1 / 2}$ so as to minimize the impact of the CCB constraint (indicated by a solid line) and tan $\beta$ $=$ (a) 3, (b) 5, (c) 10 and (d) 20. The region excluded by our $b \rightarrow s \gamma$ analysis has light shading. The region allowed by the cosmological constraint $0.1 \leqslant \Omega_{\chi} h^{2} \leqslant 0.3$, after including coannihilations, has medium shading. Dotted lines delineate the announced LEP constraint on the $\tilde{e}$ mass and the disallowed region where $m_{\tilde{\tau}_{1}}<m_{\chi}$ has dark shading. The contour $m_{\chi^{ \pm}}=102 \mathrm{GeV}$ is shown as a near-vertical dashed line in each panel. Also shown as dot-dashed lines are relevant Higgs boson mass contours.

only pure accelerator constraint on $m_{1 / 2}$ comes from the chargino mass limit. However, as we have demonstrated previously, cosmology excludes such high values of $m_{0}$, thus maintaining the importance of the Higgs boson mass bound in limiting $m_{1 / 2}$, and ultimately $m_{\chi}$ and $\tan \beta$. The mediumshaded regions in Figs. 8 and 9 show the areas in the $m_{0}, m_{1 / 2}$ plane for which the relic cosmological density falls between $0.1<\Omega h^{2}<0.3$ when co-annihilation effects are included. We note that the chargino mass constraint now essentially excludes the re-entrant parts of the dark matter density contours caused by resonant direct-channel annihilations when $m_{1 / 2} \leqslant 160 \mathrm{GeV}$, which were visible in Fig. 1 as well as Fig. 9. The dark shaded regions in Figs. 8 and 9 correspond to a charged LSP, as indicated.

We show in Fig. 10 the "tail" of the cosmological region where $m_{\chi} \sim m_{\tau_{1}}$ for $\tan \beta=3$. As can be seen in Fig. 7 of [10], the tip of this region is allowed by the CCB constraint for $\tan \beta=3,10$, and we can see in Fig. 8 that the CCB constraint is weaker for $\tan \beta=5$. We show in Fig. 10 the $m_{h}$ $=104 \mathrm{GeV}$ contour, corresponding to the 1999 bound on the Higgs boson mass after allowing for a $3 \mathrm{GeV}$ theoretical uncertainty in the prediction. We recall that the Higgs boson mass limit is even stronger for negative $\mu$. We can safely set the limit $\tan \beta>2.8$ for $\mu>0$ in the UHM. Overall, the limits we obtain on $\tan \beta$ in different LEP scenarios for both signs of $\mu$ are shown in Table II.

We now repeat the above UHM analysis for the more conservative case $\left(\mathrm{UHM}_{\text {min }}\right)$ in which we do not require stability against collapse into a CCB vacuum, and we allow $A_{0}$ to vary as far as possible, consistent with the experimental constraints on $m_{\tilde{t}_{1}}$ in particular. Figure 11 shows options for $\tan \beta$ and the sign of $\mu$ which exhibit interesting differences from the previous CCB case. For simplicity, we have chosen not to include the regions that are excluded by the $b \rightarrow s \gamma$ constraint: these turn out to be essentially independent of $A_{0}$, and hence may be taken from the corresponding panels in Fig. 8. The interesting and significant differences are in the contours of the Higgs boson mass. The Higgs boson mass is sensitive to $A_{0}$, and may be significantly lower than in the previous CCB case, with corresponding implications for the lower limits on $m_{\chi}$ and $\tan \beta$ that we quote below.

Clearly, by allowing $A_{0}$ to vary (rather than restrict its value to $-m_{1 / 2}$ ), we expect weaker bounds from the Higgs boson mass than those found when the CCB constraints were incorporated. Since the Higgs boson mass constraint was only important for lower values of $\tan \beta$, we show results only for $\tan \beta=3,5$ in Fig. 11. Whilst, for $\tan \beta=3$ and $\mu$ $<0$, the 104 and $108 \mathrm{GeV}$ Higgs boson mass contours are still to the right of the displayed region in the figure, we see that in the other cases shown, all of the contours are moved substantially to the left. In fact for $\tan \beta=5$ and $\mu>0$, the Higgs boson mass bound is no longer competitive with the chargino bound. As in the previous UHM case, we also find 

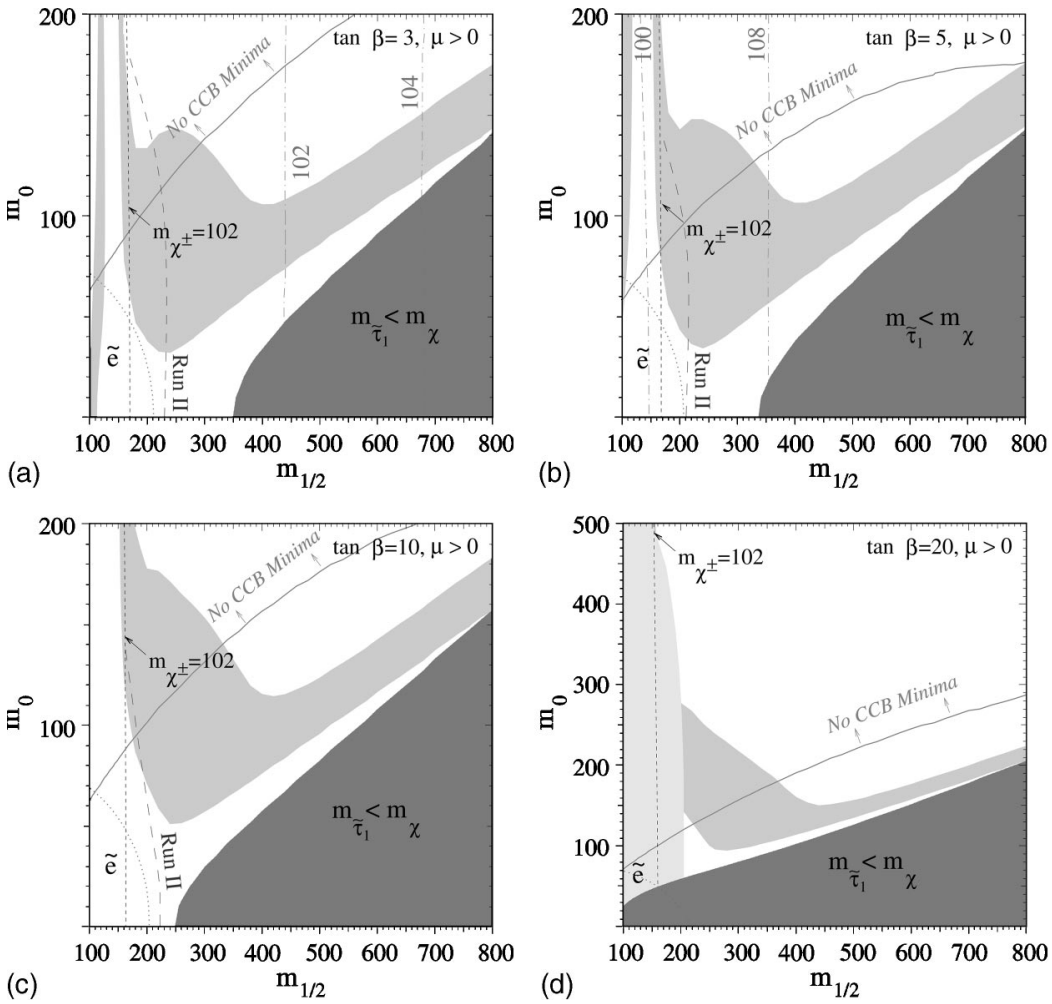

FIG. 9. The $m_{1 / 2}, m_{0}$ plane for $\mu>0, A_{0}=-m_{1 / 2}$ and $\tan \beta=$ (a) 3, (b) 5, (c) 10 and (d) 20 . The significances of the curves and shadings are the same as in Fig. 8. The light-shaded region in panel (d) is excluded by the $b \rightarrow s \gamma$ constraint. The long dashed curves in panels (a), (b) and (c) represent the anticipated limits from trilepton searches at run II of the Tevatron [2].

lower bounds on $\tan \beta$ in this case where the $\mathrm{CCB}$ constraint is relaxed, as shown in Table III for different LEP running scenarios and the two signs of $\mu$.

\section{BOUNDS FOR NON-UNIVERSAL SCALAR MASSES}

In the previous section, we have derived stringent limits on the $m_{1 / 2}, m_{0}$ plane from the absence of sparticles and Higgs bosons at LEP, assuming universality for scalar masses including the soft Higgs boson masses (UHM). These limits are particularly strong at low values of $\tan \beta$, and in

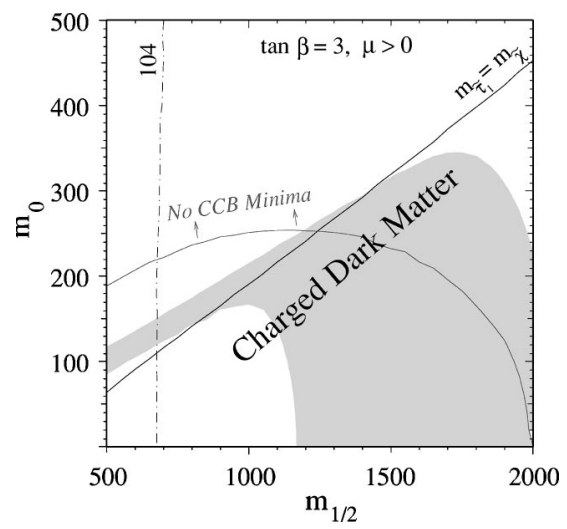

FIG. 10. An extension of the $m_{1 / 2}, m_{0}$ plane for $\mu>0, A_{0}$ $=-m_{1 / 2}$ and $\tan \beta=3$. Below the solid diagonal line, the LSP is charged and hence excluded. The absolute upper bound to $m_{1 / 2}$ is found when the shaded region drops entirely below this contour. fact exclude $\tan \beta \lesssim 2.8$. One should expect these limits to weaken when the assumption of UHM is relaxed (NUHM). In this section, we rederive the appropriate limits in the $m_{1 / 2}, m_{0}$ plane for the more general NUHM case. We now treat both $\mu$ and $m_{A}$ as independent parameters, in addition to the free parameters $m_{1 / 2}, m_{0}, A$, and $\tan \beta$ of the previous section. As indicated in Sec. II, we take $m_{A}=10 \mathrm{TeV}$, so that the Higgs boson mass limits give the most conservative bounds on $m_{1 / 2}$. As before, we restrict the values of $A$ by requiring that $m_{\tilde{t}_{1}}$ be consistent with the experimental lower limit, and $m_{\chi}>m_{\tilde{\tau}_{1}}$, as shown in Fig. 3.

Our results for the NUHM case are shown in Figs. 12 and 13. We again show the kinematical limit on the chargino mass: $m_{\chi}^{ \pm}=102$ by the near-vertical dashed line. Again, for $\mu>0$, these lines are essentially vertical, because the chargino mass is independent of $m_{0}$, apart from the effects of radiative corrections. As seen in the different panels of Fig. 12 , the lower bound on $m_{1 / 2}$ from the chargino bound increases from $m_{1 / 2}=112 \mathrm{GeV}$ to $145 \mathrm{GeV}$ as $\tan \beta$ is increased from 3 to 20 for $\mu<0$. In contrast, as seen in Fig. 13, the bound on $m_{1 / 2}$ for $\mu>0$ lies near $140 \mathrm{GeV}$ over the same

TABLE II. Limits on $\tan \beta$ imposed in the UHM by the 1999 and "realistic"' expected $2 \mathrm{~K}$ Higgs mass limits.

\begin{tabular}{ccc}
\hline \hline & 1999 & "realistic" $2 \mathrm{~K}$ \\
\hline$\mu<0$ & 3.2 & 4.0 \\
$\mu>0$ & 2.8 & 3.6 \\
\hline \hline
\end{tabular}



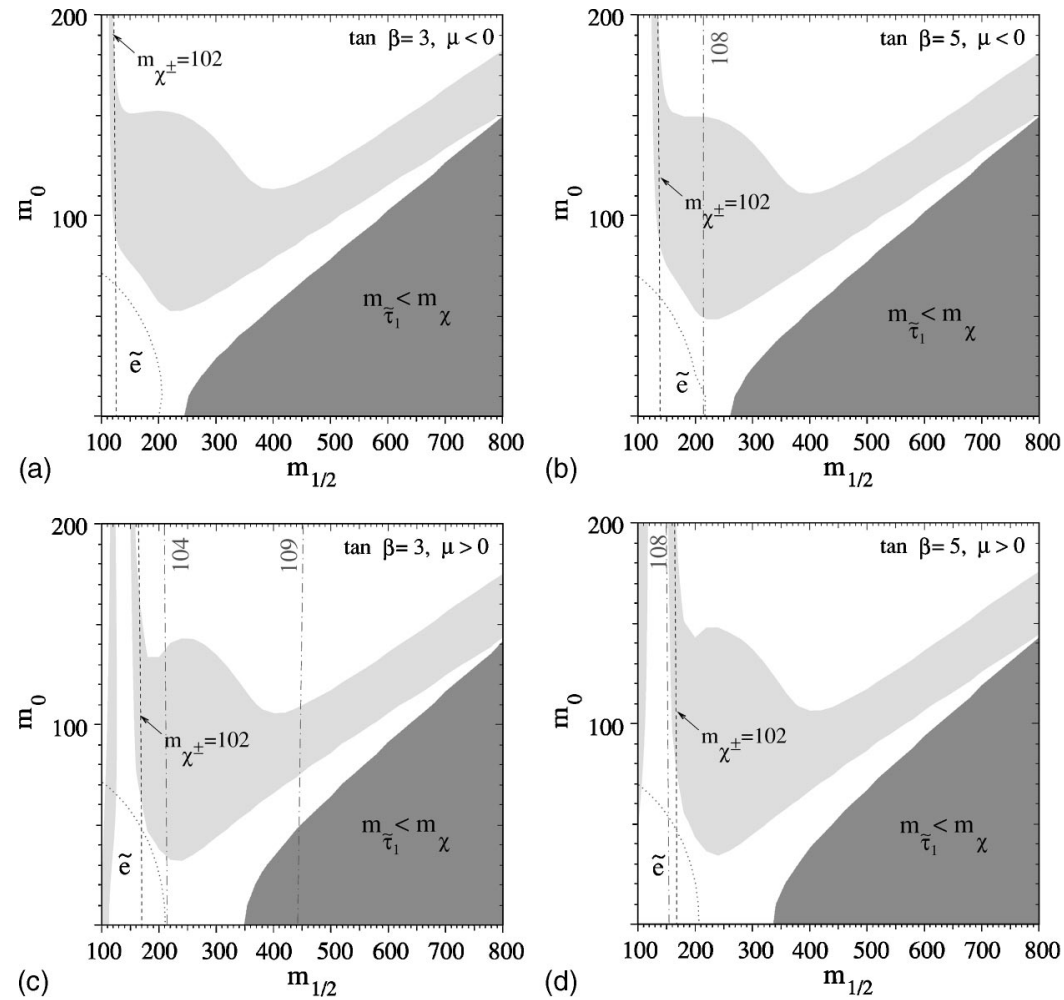

FIG. 11. The $m_{1 / 2}, m_{0}$ plane for $\mu<0$ and (a) $\tan \beta=3$, (b) $\tan \beta=5$, and $\mu>0$ and (c) $\tan \beta=3$, (d) $\tan \beta=5$, with $A_{0}$ allowed to vary, and no CCB constraint applied. For clarity, the region excluded by the $b \rightarrow s \gamma$ constraint has not been shaded: it is essentially identical to that in Fig. 8. The region allowed after including coannihilations, by the cosmological constraint $0.1 \leqslant \Omega_{\chi} h^{2} \leqslant 0.3$ has medium shading. Dotted lines delineate the announced LEP constraint on the $\tilde{e}$ mass and the disallowed region where $m_{\tilde{\tau}_{1}}<m_{\chi}$ has dark shading. The contour $m_{\chi^{ \pm}}=102 \mathrm{GeV}$ is shown as a near-vertical dashed line in each panel. Also shown as dot-dashed lines are relevant Higgs boson mass contours.

range in $\tan \beta$. As expected, the NUHM curves for the chargino mass limits always lie to the left of the UHM ones.

As in Figs. 8, 9, and 11, the shaded region corresponds to the parameter values in which it is possible to achieve 0.1 $\leqslant \Omega h^{2} \leqslant 0.3$. This region is unbounded from above, since it is possible to adjust $\mu$ to insure an acceptable relic density even if the sfermion masses are large. This is because by lowering $\mu$, the LSP can become a mixed state (rather than an almost pure $b$-ino) and annihilation channels via $Z^{0}$-exchange open up.

We also show in Figs. 12a (b) and 13a (b) the NUHM contours for Higgs boson masses of 104 (100) and 109 (108) $\mathrm{GeV}$, the 1999 and $2 \mathrm{~K}$ "realistic" bounds for $\tan \beta=3$ $(\tan \beta=5)$. For $\tan \beta=5$ and $\mu>0$, the $100 \mathrm{GeV}$ contour is to the left of the chargino mass contour and is not shown. We recall that, in the NUHM case, there are no unique Higgs boson mass contours in the $m_{0}, m_{1 / 2}$ plane, due to the free-

TABLE III. Limits on $\tan \beta$ imposed in the UHM by the 1999 and "realistic" expected $2 \mathrm{~K}$ Higgs mass limits, relaxing the requirement that there be no CCB vacuum.

\begin{tabular}{ccc}
\hline \hline & 1999 & "realistic" $2 \mathrm{~K}$ \\
\hline$\mu<0$ & 2.7 & 3.1 \\
$\mu>0$ & 2.2 & 2.7 \\
\hline \hline
\end{tabular}

dom in choosing $\mu, m_{A}$ and $A_{0}$. The contours shown correspond to parameter choices giving the weakest bound.

Because the Higgs boson mass bound is weakest for relatively large values of $\mu$ in the NUHM case (implying that the neutralino is a $b$-ino), the relic LSP density increases with $m_{0}$ as one moves upward along the Higgs boson mass contours and the sfermion masses increase. In some cases, e.g., that in Fig. 13a, the relic density along the $104 \mathrm{GeV}$ contour exceeds 0.3 at about $m_{0}=140 \mathrm{GeV}$. At higher values of $m_{0}$, the value of $m_{1 / 2}$ must be increased to remain consistent with both the Higgs boson mass limits and cosmology. This adjustment is shown by the dotted curve to the right of this contour. Similar behavior was seen in [21]. For the other Higgs boson mass contours, either the shift (in $m_{1 / 2}$ ) is insignificant at $m_{0} \leqslant 200 \mathrm{GeV}$, or the relic density does not exceed $\Omega h^{2}=0.3$ for $m_{0} \leqslant 200 \mathrm{GeV}$. For $\mu<0$, the relic density is never saturated for the values of $m_{0}$ shown. As before, for $\tan \beta=10$ and 20 , the chargino bound is always stronger than the Higgs boson mass bound.

\section{HIGGSINO DARK MATTER}

We now turn to the question whether there is any room left for Higgsino dark matter. We update the analysis of [3], including the improved experimental limits discussed above, and we explore the sensitivity of our conclusions to the pos- 

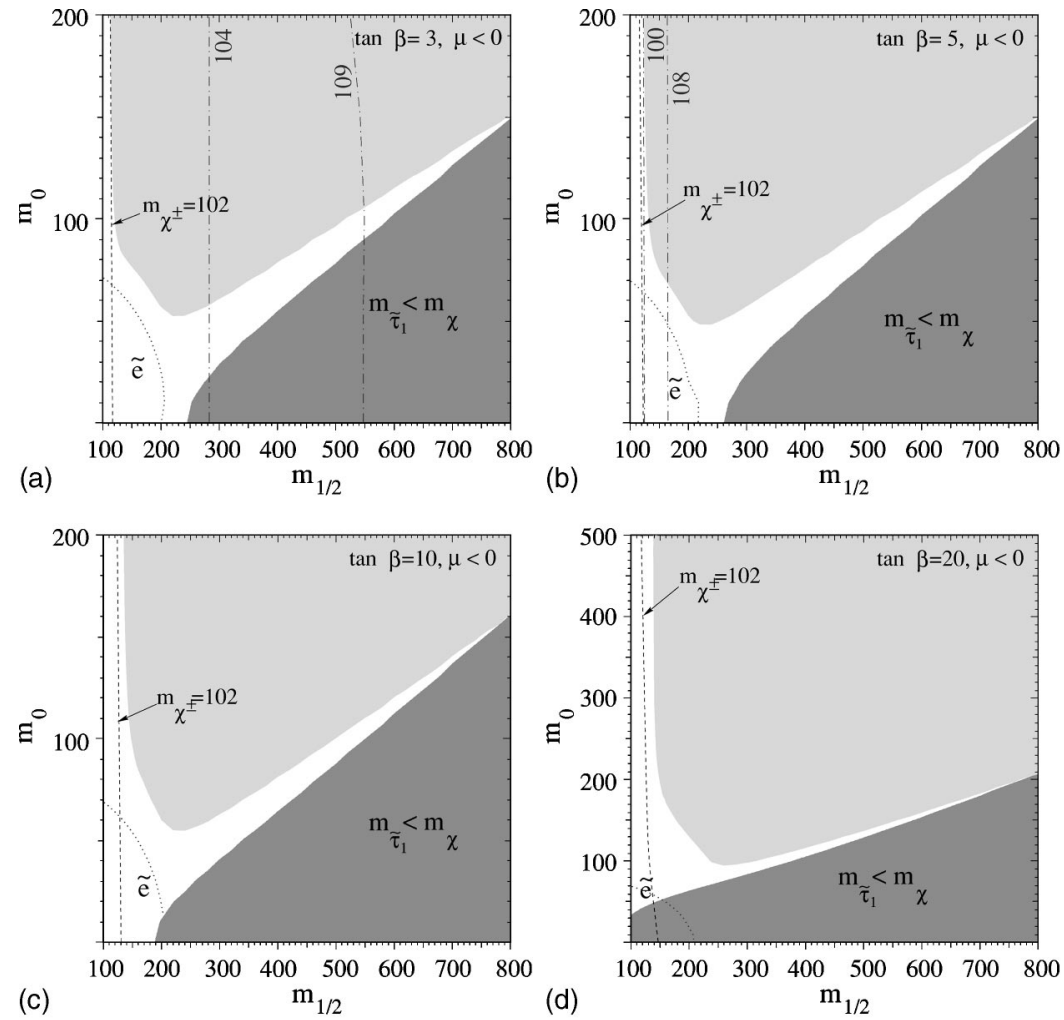

FIG. 12. The $m_{1 / 2}, m_{0}$ plane for $\mu<0$ in the NUHM case, for $\tan \beta=$ (a) 3, (b) 5, (c) 10 and (d) 20. The region allowed after including coannihilations, by the cosmological constraint $0.1 \leqslant \Omega_{\chi} h^{2} \leqslant 0.3$ is shown shaded. Dotted lines delineate the announced LEP constraint on the $\tilde{e}$ mass and the disallowed region where $m_{\tau_{1}}<m_{\chi}$ has dark shading. The contours $m_{\chi^{ \pm}}=102 \mathrm{GeV}$ are shown as near-vertical dashed lines. Also shown as near-vertical dot-dashed lines are Higgs boson mass contours.

sible range of LSP relic density. We begin by reviewing briefly the analysis of [3].

We recall that a general neutralino is a linear combination of the Higgsinos and neutral gauginos, $\quad \chi=\beta \widetilde{B}+\alpha \widetilde{W}_{3}$ $+\gamma \widetilde{H}_{1}+\delta \widetilde{H}_{2}$. In this notation, the Higgsino purity is defined to be $p=\sqrt{\gamma^{2}+\delta^{2}}$, a state that is half gaugino and half Higgsino has Higgsino purity $1 / \sqrt{2}$, and, as in [3], we take as our working definition that a neutralino is a "pure" Higgsino if $p>0.9$, even though such a state already has a sizeable gaugino fraction: $\sqrt{\alpha^{2}+\beta^{2}} \sim 0.44$. The lightest neutralino tends to be Higgsino-like if $\mu<M_{2} / 2$ and gauginolike when $\mu>M_{2} / 2$. This was already shown in Fig. 1, where we plot as hashed lines contours of Higgsino purity in the $\mu, m_{1 / 2}$ plane for $\tan \beta=3$. We have also plotted thin solid contours for $\Omega_{\chi} h^{2}=0.025,0.1$ and 0.3 , thick solid lines corresponding to $m_{\chi^{ \pm}}=100 \mathrm{GeV}$, and dot-dashed Higgs boson mass contours. In this illustration we have taken $m_{A}$ $=1 \mathrm{TeV}$ and $m_{0}=100 \mathrm{GeV}$. It is apparent that the bulk of the cosmological region with $0.1 \leqslant \Omega_{\chi} h^{2} \leqslant 0.3$ has larger $|\mu|$ (for given $M_{2}$ ) than do the Higgsino purity contours, indicating that LSP dark matter is generically a gaugino: in these regions, it is mainly a Bino. There are, however, small regions at smaller $|\mu|$ (for given $M_{2}$ ), where the LSP is mainly a Higgsino. However, as can be seen in Fig. 1, this Higgsino possibility is under severe pressure from several LEP constraints, including the chargino, $\chi \chi^{\prime}$ and Higgs searches, and it is the possible exclusion of the Higgsino dark matter regions we explore in this section.

Accordingly, we now focus in more detail on the Higgsino regions, as illustrated in Figs. 14 and 15, where detailed views of the Higgsino parts of the $\mu, M_{2}$ plane are shown for $\tan \beta=2,3,5$ and 10. Consider in particular Fig. $14 \mathrm{a}$, for $\tan \beta=2$ and $\mu<0$. Here we have taken $m_{A}$ $=10 \mathrm{TeV}$ to minimize the effect of the Higgs boson mass limit, and $m_{0}=1 \mathrm{TeV}$ to maximize the neutralino relic density. In contrast to Fig. 1, we have also adjusted $A_{t}$ to maximize the Higgs boson mass and produce the weakest Higgs constraint. The hashed, dot-dashed, thin solid and dark thick solid contours are as in Fig. 1. We plot as a dashed line the current chargino mass limit. We also show as two solid contours the most recent 1999 LEP 2 bounds on the summed visible cross section for associated neutralino production $\sigma\left(e^{+} e^{-} \rightarrow \chi_{i} \chi_{j}\right)_{\text {vis }}$ and our "realistic"' estimate for the final $2 \mathrm{~K}$ bounds (see Table I). We recall that the associated production bounds are more constraining for smaller values of the scalar masses. ${ }^{4}$ It is evident that the entire region with $\Omega_{\chi} h^{2}>0.1$ and Higgsino purity $p>0.9$ is excluded by the current experimental limits, for this value of $\tan \beta$.

\footnotetext{
${ }^{4}$ For the sake of exposition, we forget for the moment that, at this low value of $\tan \beta$, the entire displayed region has a Higgs boson mass less than $106 \mathrm{GeV}$ and can be excluded on this basis alone.
} 

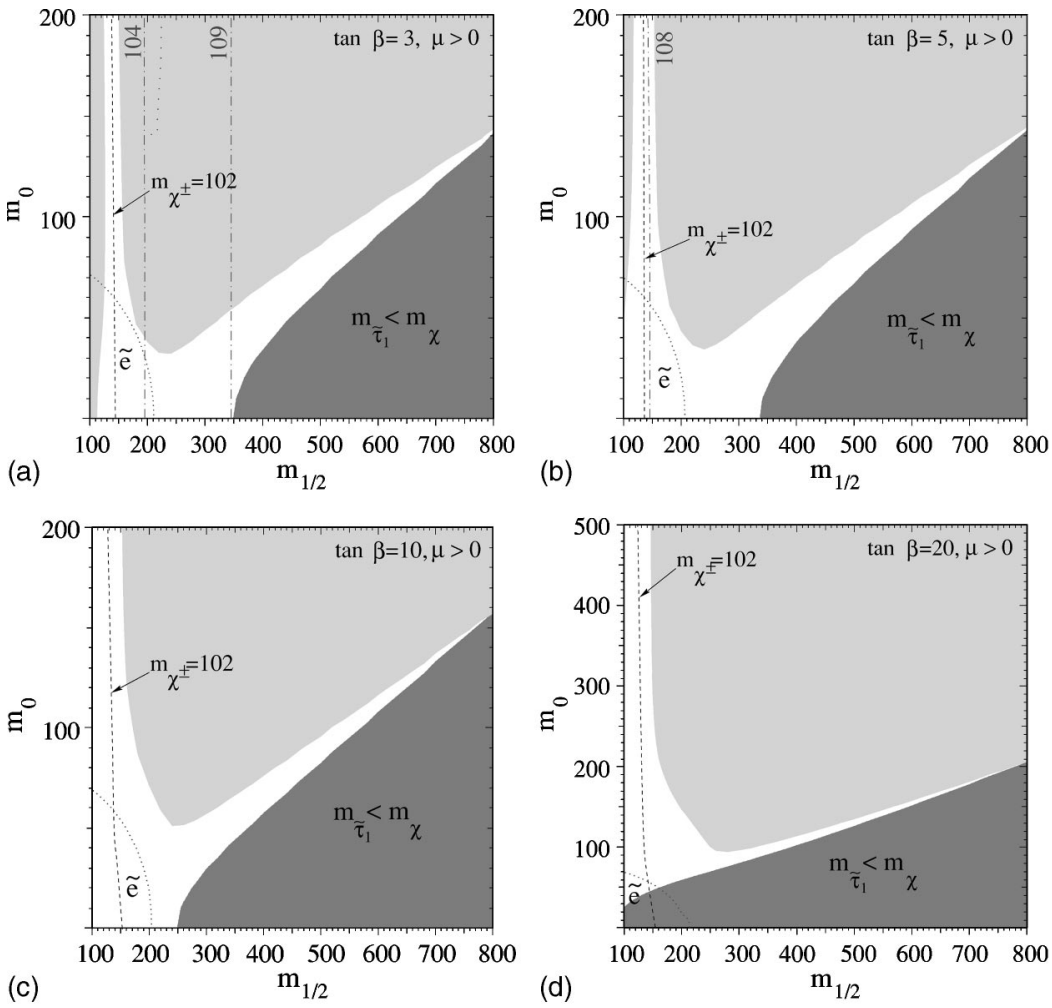

FIG. 13. The $m_{1 / 2}, m_{0}$ plane for $\mu>0$ in the NUHM case, for $\tan \beta=$ (a) 3, (b) 5, (c) 10 and (d) 20. The significances of the curves and shadings are the same as in Fig. 12. The dotted extension of the $m_{h}=104 \mathrm{GeV}$ contour corresponds to the shift in the Higgs boson contour when the cosmological limit on the relic density is imposed [visible only in (a)].

The general shape of the $\Omega_{x} h^{2}$ contours can be understood as follows. As one moves to large values of $M_{2}$, the neutralino becomes more pure Higgsino, which leads to an approximate three-way mass degeneracy between the lightest and next-to-lightest neutralinos $\chi, \chi_{2}$ and the lightest chargino $\chi^{ \pm}$. Since the $\chi_{2}$ and $\chi^{ \pm}$are therefore abundant at the time when the $\chi$ freezes out of chemical equilibrium in the early Universe [35], their coannihilations with the $\chi$ act to bind the $\chi$ more tightly in chemical equilibrium with the thermal bath, and delay the freeze-out of the $\chi$ relic density. Since the $\chi, \chi_{2}$ and $\chi^{ \pm}$annihilate very efficiently, this greatly reduces the relic density of neutralinos for larger values of $M_{2}$ [36]. We have included the one-loop radiative corrections to the chargino and neutralino masses, which can significantly affect both the experimental chargino limits and the neutralino relic density, when the chargino and neutralino are closely degenerate. Whenever the mass degeneracy is sufficiently tight to affect the chargino bounds, coannihilation suppresses the relic density to very small values, below those of cosmological interest [3].

Similarly, as one moves to larger values of $|\mu|$, the mass of the $\chi$ increases, until $m_{\chi}>m_{W}$. At this point, the $\chi$ can annihilate efficiently into $W$ pairs, and the relic density drops dramatically as one crosses this threshold. ${ }^{5}$ The light solid

\footnotetext{
${ }^{5}$ Sub-threshold annihilation into $W$ pairs smoothes out this sudden drop [35], and shifts the left edge of the $\Omega_{\chi} h^{2}=0.1$ contour a few $\mathrm{GeV}$ to the right.
}

contour corresponds to $m_{\chi}=m_{W}$, and the falloff of the relic density above this threshold is evident. It is the dramatic decrease in $\Omega_{\chi} h^{2}$ for $m_{\chi}>m_{W}$ that not only excludes Higgsino dark matter, but also implies that we are not very dependent on our default choice of lower relic density cutoff: $\Omega_{\chi} h^{2}>0.1$. In fact, one cannot even supply enough neutralinos to provide the galactic dark matter: $\Omega_{\chi} h^{2} \sim 0.025$ above the $W$ threshold, while still satisfying the experimental constraints. We see the same effect in all four panels of Figs. 14, and, although we only display four values of $\tan \beta$, we have verified that this is true for all $\tan \beta$.

The situation is similar for $\mu>0$, as seen in Fig. 15. In this case, the dominant experimental constraint comes from the chargino limits, which are shown as dashed lines for both 1999 and the "realistic" $2 \mathrm{~K}$ scenario, whereas we plot only the 1999 contour for $e^{+} e^{-} \rightarrow \chi_{i} \chi_{j}$. The chargino constraints alone exclude $\Omega_{\chi} h^{2}>0.025$ for a Higgsino-like neutralino. In all cases, an interesting amount of cold dark matter is possible only if $p^{2} \leqslant 0.7$, i.e., the LSP is either predominantly a gaugino or a strongly mixed state. We conclude that $a$ predominantly Higgsino state cannot provide a substantial component of the dark matter.

This conclusion is robust with respect to variations in the other sparticle masses. The sfermion masses have already been taken large enough for the contribution to neutralino annihilation from sfermion exchange to be negligible, and, as already noted, lighter sfermions yield tighter constraints from associated neutralino production. The experimental chargino limits fall below the kinematic limit when the 

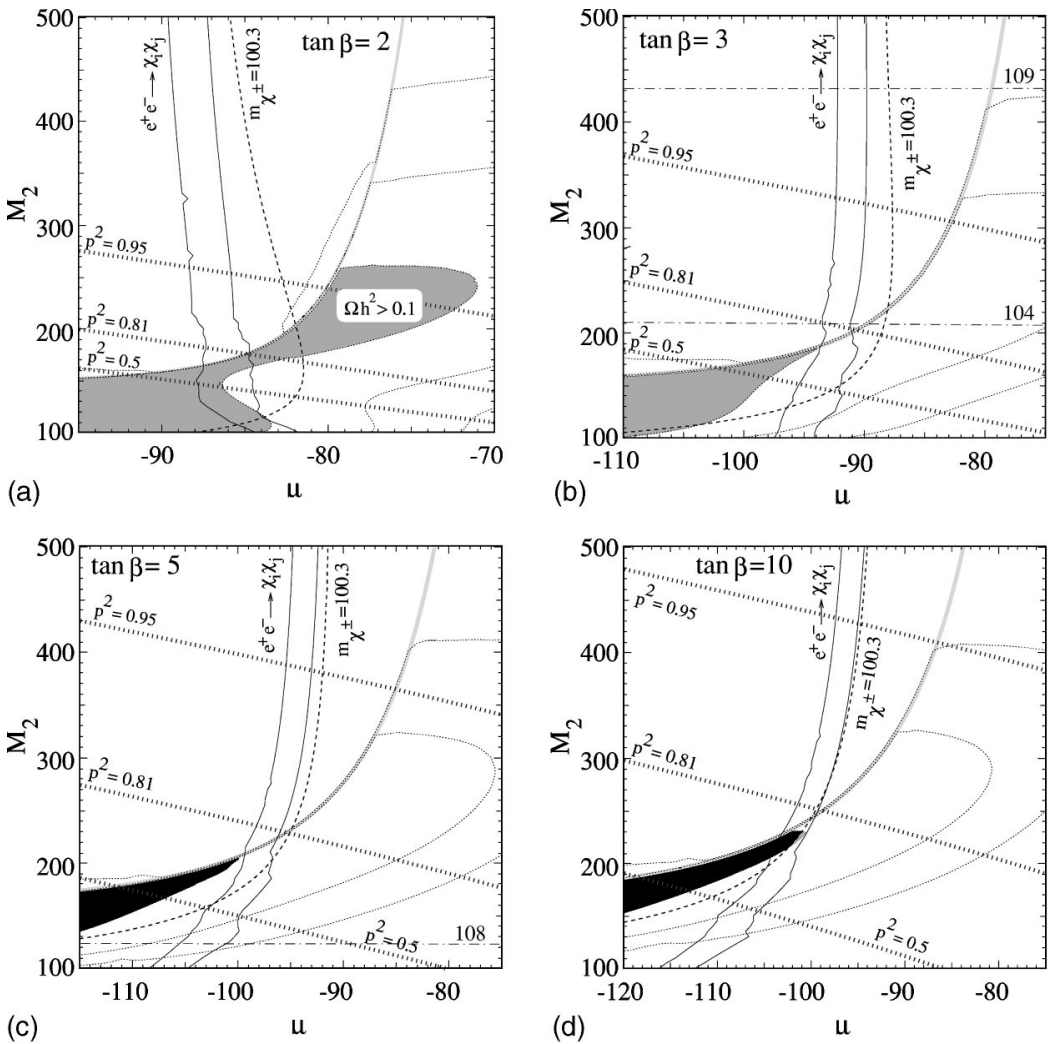

FIG. 14. Small regions of the $\mu, M_{2}$ plane for $\tan \beta=2,3,5,10$ and $m_{A}=10 \mathrm{TeV}$. The thin solid lines are contours for $\Omega_{\chi} h^{2}$ $\geqslant 0.1,0.05,0.025$, and the light-shaded region is cosmologically preferred. The dashed (light solid) lines correspond to $m_{\chi^{ \pm}}=100.3 \mathrm{GeV}$ $\left(m_{\chi}=m_{W}\right)$, dot-dashed horizontal lines correspond to the indicated Higgs boson masses, and the two darker near-vertical solid contours indicate the current neutralino associated production bound $\sigma\left(e^{+} e^{-} \rightarrow \chi \chi_{2}, \ldots\right)$ vis and our estimate of the "realistic" final bound (see Table I). Hashed contours represent Higgsino purity. In panel (a) [(d)], the Higgs boson mass is everywhere less than $106 \mathrm{GeV}$ [greater than $109 \mathrm{GeV}]$. The dark-shaded regions in panels (c) and (d) are those surviving all the constraints.

sneutrino is closely degenerate with the chargino: however, again, a light sneutrino enhances the associated neutralino production limits, so this also provides no loophole. A cosmologically interesting relic density can therefore only be achieved by either heavily mixed or pure gaugino neutralino states.

\section{LIMITS FROM THE TEVATRON COLLIDER}

The limits on the MSUGRA (UHM) parameter space from run I of the Tevatron are summarized in [2], and we have already made use of their lower limits on stop masses. Two other D0 limits are reported in [2]: one is for squark and gluino jets and missing energy, and the other is for dilepton events. The former analysis is for $\tan \beta=2$, and hence is not directly comparable with our plots. The analysis also assumes $A_{0}=0$ and $\mu<0$, and yields a lower limit

$$
m_{1 / 2}>100 \text { to } 50 \mathrm{GeV}
$$

for $m_{0}$ between 0 and $300 \mathrm{GeV}$. The dilepton analysis in [2] has been performed for several values of $\tan \beta$ including the values 3 and 5 studied in this paper. Again for $A_{0}=0$ and $\mu<0$, the lower limit

$$
m_{1 / 2}>60 \text { to } 40 \mathrm{GeV}
$$

was found for $m_{0}$ between 0 and $300 \mathrm{GeV}$. These Tevatron run I limits do not constrain the MSSM parameter space as strongly as do the chargino and Higgs limits from LEP II, unless scalar mass universality between squarks and sleptons is relaxed. We have therefore not displayed the run I bounds in Figs. 8 and 9.

On the other hand, run II of the Tevatron, scheduled to begin in 2001, will impose strict new limits at low $m_{1 / 2}$. The dominant experimental constraint is expected to come from searches for trilepton signatures of $\chi_{1}^{ \pm} \chi_{2}^{0}$ production [37,2]. We display in Fig. 9 as long-dashed contours the anticipated reach of the upgraded Tevatron in this channel, assuming $2 \mathrm{fb}^{-1}$ integrated luminosity. We have taken the published curves from [2], which does not display results for $\tan \beta$ $=20$ or $\mu<0 .{ }^{6}$ The bounds are tightest at low $m_{0}$, where they cut into the cosmological region as shown. At $\tan \beta$ $\gtrsim 3$, the run II curves bend over and intersect the LEP2 chargino bound within the cosmological region. Their most significant impact is for low values of $\tan \beta$. However, since the LEP II Higgs bounds dominate at low $\tan \beta$, the trilepton

\footnotetext{
${ }^{6}$ The $\tan \beta=3,10$ run II curves and the $\tan \beta=5$ run II curve in Fig. 9 come from separate analyses and reflect slightly different confidence levels, $99 \%$ and $3 \sigma$, respectively.
} 

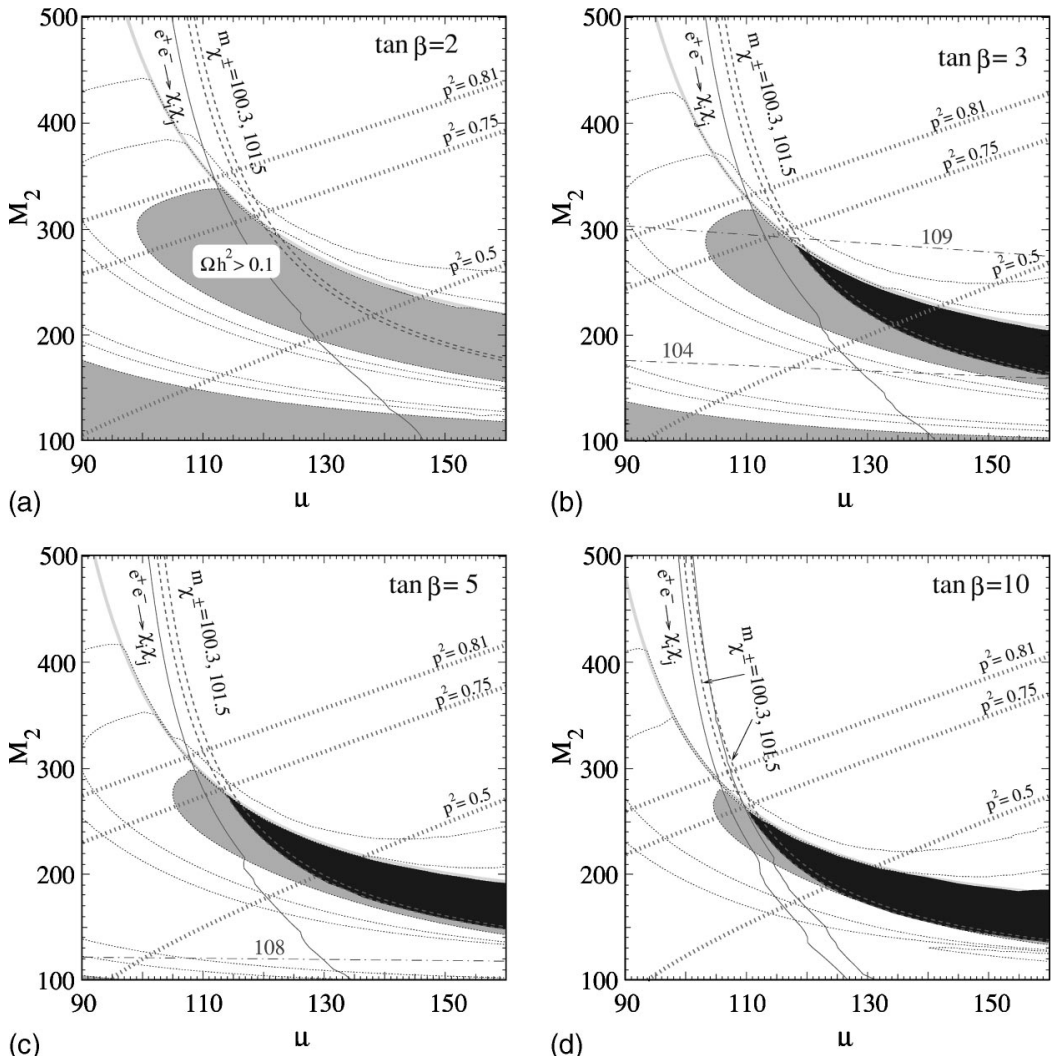

FIG. 15. As in Fig. 14, for $m u>0$.

analysis at the Tevatron run II will therefore not increase the absolute lower bound on the neutralino mass given by our analysis in this region. However, if the Tevatron is able to improve the LEP Higgs bound, this could raise substantially the neutralino limit at low $\tan \beta$. The Higgs bound is no longer important for $\tan \beta=10$, but in this case the Tevatron limit bites away less of the region favored by cosmology.

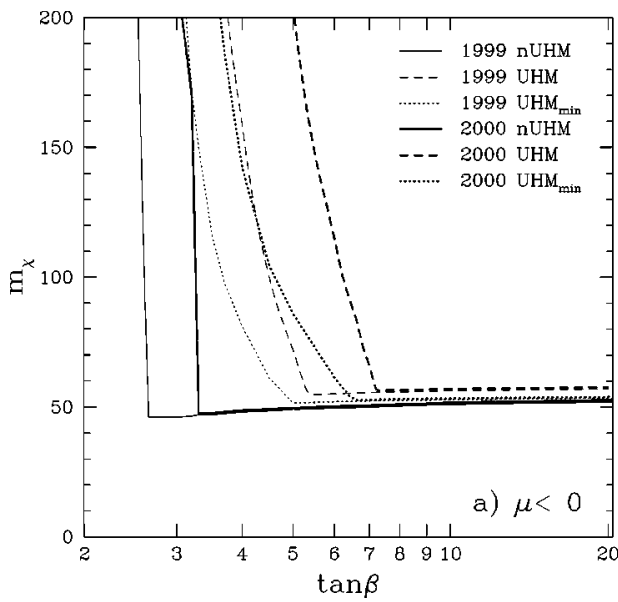

\section{SUMMARY AND CONCLUSIONS}

One of our principal goals in this paper has been to obtain strengthened lower limits on the neutralino mass, combining the latest LEP data with the cosmological dark matter requirement $0.1<\Omega_{\chi} h^{2}<0.3$. We summarize our limits in Figs. 16, under various different assumptions: universal

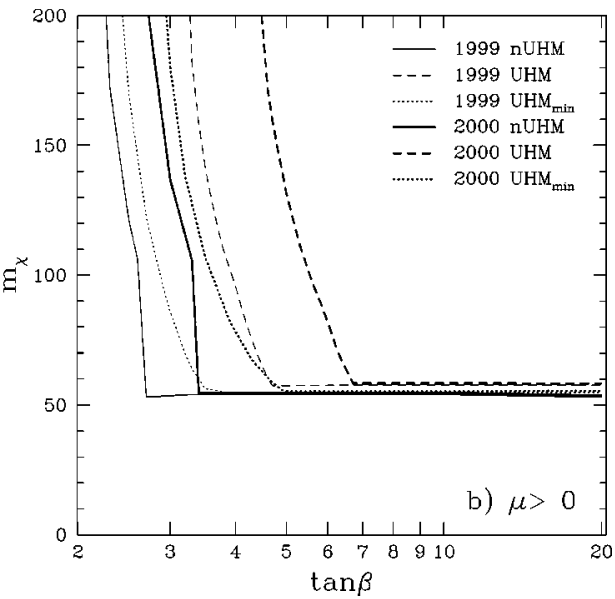

FIG. 16. Lower limits on the neutralino mass $m_{\chi}$ as functions of $\tan \beta$ for (a) $\mu<0$ and (b) $\mu>0$. The curves correspond to the final 1999 LEP results (thin lines) and our "realistic" expectations for the $2 \mathrm{~K}$ LEP run (thick lines). We show the UHM case with $A_{0}=-m_{1 / 2}$ to avoid CCB minima (dashed curves): these are the strongest constraints. We also show (dotted lines) the more general $\mathrm{UHM}_{\min }$ case where $A_{0}$ is left free, and we do not require the absence of CCB vacua. We also display additionally the NUHM case, which is the most conservative and allows both $\mu$ and $m_{A}$ to be free in addition to $A_{0}$. Note that the $b \rightarrow s \gamma$ constraints have not been applied in these figures. For the UHM cases, they would effectively exclude portions of the $\tan \beta$, low $m_{\chi}$ region. The precise regions excluded by $b \rightarrow s \gamma$ can be gleaned from Figs. 8 and 9 by noting that $m_{\chi} \sim 0.4 m_{1 / 2}$. 


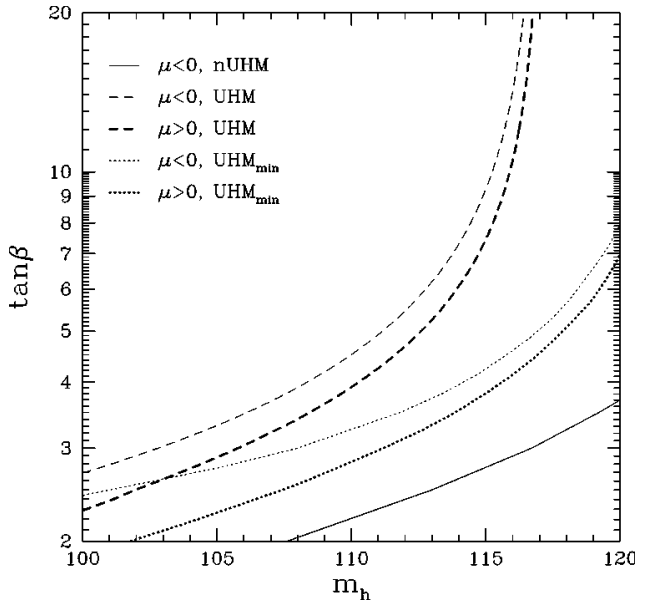

FIG. 17. Lower limit on $\tan \beta$ imposed by the experimental and cosmological constraints, as a function of the experimental Higgs boson mass limit. The UHM, UHM $\mathrm{Uin}_{\text {in }}$ and NUHM labels are as in Fig. 16. The $\mu>0$ curve in the NUHM case is very similar to the $\mu<0$ curve.

(UHM) or non-universal (NUHM) soft supersymmetrybreaking scalar masses for Higgs bosons and (in the former case) whether one requires the present vacuum to be stable against transition to a charge- and color-breaking (CCB) vacuum or not $\left(\mathrm{UHM}_{\min }\right)$. Also, we give limits both for the available 1999 LEP data and with a "realistic" assessment of the likely sensitivity of data to be taken in $2 \mathrm{~K}$.

In all cases, for both positive and negative $\mu$, the lower limits on $m_{\chi}$ are relatively insensitive to $\tan \beta$ at large $\tan \beta$. Here they are determined by the LEP chargino bound, as the LEP Higgs boson mass bound is weaker than the chargino bound at large $\tan \beta$. In fact, in the two UHM cases shown, the points at which the limiting curves bend upward, as one decreases $\tan \beta$, are precisely the points at which the Higgs boson mass bound becomes more stringent than the chargino bound. In the UHM cases, the neutralino mass limits are strong at intermediate values of $\tan \beta \simeq 4-7$ because, as discussed earlier, the cosmological bound on the relic density prohibits going to large values of $m_{0}$, and ensures that the Higgs bound places a strong constraint. Below this break point, the lower limit on $m_{\chi}$ increases rapidly with decreasing $\tan \beta$. Above this break point, the limit on $m_{\chi}$ is relatively insensitive to the additional theoretical assumptions made, such as UHM vs UHM min $_{\text {in }}$ or NUHM. However, in the NUHM cases, because one can increase $m_{0}$ sufficiently to weaken the Higgs boson mass bound, the break point occurs at a lower value of $\tan \beta$. To go to lower values of $\tan \beta$ then requires a substantial increase in $m_{\chi}$.

In the UHM cases with and without the restriction forbidding CCB vacua, the lower limit on the lightest MSSM Higgs boson mass, in particular, implies lower limits on $\tan \beta$ which are plotted in Fig. 17. The limits are somewhat stricter for $\mu<0$ than for $\mu>0$, whether (UHM) or not $\left(\mathrm{UHM}_{\text {min }}\right)$
TABLE IV. Limits on $\tan \beta$, assuming the "optimistic" $2 \mathrm{~K}$ energies and luminosities (9).

\begin{tabular}{cccc}
\hline \hline & UHM & UHM $_{\min }$ & NUHM \\
\hline$\mu<0$ & 4.7 & 3.4 & 2.3 \\
$\mu>0$ & 4.2 & 3.0 & 2.3 \\
\hline
\end{tabular}

one requires the absence of CCB vacua. Indeed, Fig. 17 will enable the appropriate conclusion to be drawn from whatever lower limit on the Higgs boson mass LEP eventually provides. We recall that the existing Higgs boson mass calculations in the MSSM are believed to be accurate to about 3 $\mathrm{GeV}$. Therefore in computing the bounds on $\tan \beta$ for Tables II and III, for example, we have conservatively shifted the exclusion curves of Fig. 6 by $3 \mathrm{GeV}$ to the left before reading the values of $\tan \beta$ off of Fig. 17. We also show in Fig. 17 the lower bound on $\tan \beta$ obtained in the NUHM, which is significantly weaker than in the UHM cases, and essentially independent of the sign of $\mu$.

If LEP does achieve the "optimistic" $2 \mathrm{~K}$ energies and luminosities (9), the above constraints will be somewhat tighter. The horizontal segments of Fig. 16, corresponding to the chargino limits, increase by a fraction of a GeV; the vertical branches move to the right, intersecting the horizontal segments at $\tan \beta=8$ (7.5) for $\mu<0(\mu>0)$. And lastly, the lower limits on $\tan \beta$ improve to the results given in Table IV.

In many respects, LEP has provided the most stringent constraints on the parameters of the MSSM. This is true, in particular, for its lower limits on the Higgs boson mass, and the chargino, neutralino and slepton constraints from LEP compare favorably with the Tevatron bounds on squark and gluino masses, once the different mass renormalizations of electroweakly- and strongly-interacting sparticles are taken into account. As we have shown, the present LEP data may be combined to set interesting lower bounds on the lightest neutralino mass, in particular if it is assumed to constitute the dark matter favored by astrophysics and cosmology, and on $\tan \beta$. It may well be that these lower limits will be further strengthened by the LEP run during 2000, as we have discussed in this paper. However, this would be the pessimistic scenario. There is still a chance that sparticles or the Higgs boson may turn up this year, in which case we would be delighted to see our bounds superseded. LEP may not yet have discovered supersymmetry, but it certainly deserves to.

\section{ACKNOWLEDGMENTS}

We would like to thank P. Gambino and C. Kao and especially M. Schmitt and M. Spiropulu for useful discussions. This work was supported in part by DOE grant DE-FG0294ER-40823. The work of T.F. was supported in part by DOE grant DE-FG02-95ER-40896 and in part by the University of Wisconsin Research Committee with funds granted by the Wisconsin Alumni Research Foundation. 
[1] See the LEP Joint Supersymmetry Working Group URL: http://lepsusy.web.cern.ch/lepsusy/ Welcome.html.

[2] See Tevatron SUGRA Working Group Collaboration, S. Abel et al., hep-ph/0003154, and references therein.

[3] J. Ellis, T. Falk, G. Ganis, K. A. Olive, and M. Schmitt, Phys. Rev. D 58, 095002 (1998).

[4] D. M. Pierce, J. A. Bagger, K. Matchev, and R. Zhang, Nucl. Phys. B491, 3 (1997).

[5] CLEO Collaboration, M. S. Alam et al., Phys. Rev. Lett. 74, 2885 (1995); S. Ahmed et al., CLEO CONF 99-10.

[6] ALEPH Collaboration, R. Barate et al., Phys. Lett. B 429, 169 (1998).

[7] J. Ellis, J. S. Hagelin, D. V. Nanopoulos, K. A. Olive, and M. Srednicki, Nucl. Phys. B238, 453 (1984).

[8] N. Bahcall, J. P. Ostriker, S. Perlmutter, and P. J. Steinhardt, Science 284, 1481 (1999).

[9] W. L. Freedman, astro-ph/9909076.

[10] J. Ellis, T. Falk, and K. A. Olive, Phys. Lett. B 444, 367 (1998); J. Ellis, T. Falk, K. A. Olive, and M. Srednicki, Astropart. Phys. 13, 181 (2000).

[11] For recent analyses taking these effects into account, see A. B. Lahanas, D. V. Nanopoulos, and V. C. Spanos, Phys. Lett. B 464, 213 (1999); Phys. Rev. D 62, 023515 (2000); M. E. Gomez, G. Lazarides, and C. Pallis, ibid. 61, 123512 (2000); hep-ph/0004028.

[12] J. A. Casas, A. Lleyda, and C. Munoz, Nucl. Phys. B471, 3 (1996); H. Baer, M. Brhlik, and D. Castano, Phys. Rev. D 54, 6944 (1996); S. Abel and T. Falk, Phys. Lett. B 444, 427 (1998)

[13] ALEPH, DELPHI, L3 and OPAL Collaborations, "Searches for Higgs bosons: Preliminary combined results using LEP data collected at energies up to $202 \mathrm{GeV}$, ALEPH 2000-028 CONF 2000-023, DELPHI 2000-050 CONF 365, L3 note 2525, OPAL Technical Note TN546, March 2000.

[14] S. P. Martin and M. T. Vaughn, Phys. Rev. D 50, 2282 (1994).

[15] K. Inoue, A. Kakuto, H. Komatsu, and S. Takeshita, Prog. Theor. Phys. 68, 927 (1982); M. Drees and M. M. Nojiri, Nucl. Phys. B369, 54 (1992); W. de Boer, R. Ehret, and D. I. Kazakov, Z. Phys. C 67, 647 (1995).

[16] V. Barger, M. S. Berger, and P. Ohmann, Phys. Rev. D 49, 4908 (1994).

[17] A. Brignole, L. E. Ibanez, and C. Munoz, Nucl. Phys. B422, 125 (1994).

[18] M. Carena, M. Olechowski, S. Pokorski, and C. E. M. Wagner, Nucl. Phys. B426, 269 (1994), and references therein.

[19] M. Carena, J. Ellis, S. Lola, and C. E. Wagner, Eur. Phys. J. C 12, 507 (2000).

[20] P. H. Chankowski, J. Ellis, M. Olechowski, and S. Pokorski, Nucl. Phys. B544, 39 (1999); P. H. Chankowski, J. Ellis, K. A.
Olive, and S. Pokorski, Phys. Lett. B 452, 28 (1999), and references therein.

[21] J. Ellis, T. Falk, K. A. Olive, and M. Schmitt, Phys. Lett. B 413, 355 (1997).

[22] ALEPH Collaboration, ALEPH 2000-021 CONF 2000-018, contribution to 2000 Winter Conferences.

[23] LEP Supersymmetry Working Group, ALEPH, DELPHI, L3 and OPAL Collaborations, note LEPSUSYWG/99-02.1.

[24] CDF Collaboration, T. Affolder et al., FERMILAB-PUB-99/ 311-E.

[25] M. Ciuchini, G. Degrassi, P. Gambino, and G. F. Giudice, Nucl. Phys. B527, 21 (1998); P. Ciafaloni, A. Romanino, and A. Strumia, ibid. B524, 361 (1998); F. Borzumati and C. Greub, Phys. Rev. D 58, 074004 (1998).

[26] R. Barbieri and G. F. Giudice, Phys. Lett. B 309, 86 (1993).

[27] For a review, see M. Misiak, S. Pokorski, and J. Rosiek, in Heavy Flavours II, edited by A. J. Buras and M. Lindner (World Scientific, Singapore, 1998), hep-ph/9703442.

[28] M. Ciuchini, G. Degrassi, P. Gambino, and G. F. Giudice, Nucl. Phys. B534, 3 (1998).

[29] LEP and SLD experiments, "A Combination of Preliminary Electroweak Measurements and Constraints on the Standard Model,"' CERN EP/2000-016.

[30] Particle Data Group, C. Caso et al., Eur. Phys. J. C 3, 1 (1998).

[31] A. L. Kagan and M. Neubert, Eur. Phys. J. C 7, 5 (1999).

[32] Presentations at the open LEPC session, 2000: ALEPH Collaboration, P. Dornan, http://alephwww.cern.ch/ALPUB/ seminar/lepc_mar2000/lepc2000.pdf; DELPHI Collaboration, C. Mariotti, http://delphiwww.cern.ch/ $/$ pubxx/www/delsec/ talks/LEPCmar2000/chiara000307.pdf; L3 Collaboration, M. Grünewald, http://13www.cern.ch/conferences/ps/Grunewald _LEPC_March2000.ps.gz; OPAL Collaboration, R. McPherson, http://www1.cern.ch/Opal/talks/mcpherson_lepc00.ps.gz.

[33] P. Janot, Proceedings of the IX Chamonix Workshop on SPS \& LEP Performance, CERN-SL-99-007 DI.

[34] M. Carena, S. Heinemeyer, C. E. Wagner, and G. Weiglein, hep-ph/9912223; M. Carena, H. E. Haber, S. Heinemeyer, W. Hollik, C. E. Wagner, and G. Weiglein, Nucl. Phys. B580, 29 (2000).

[35] K. Griest and D. Seckel, Phys. Rev. D 43, 3191 (1991); P. Gondolo and G. Gelmini, Nucl. Phys. B360, 145 (1991).

[36] S. Mizuta and M. Yamaguchi, Phys. Lett. B 298, 120 (1993); M. Drees, M. M. Nojiri, D. P. Roy, and Y. Yamada, Phys. Rev. D 56, 276 (1997).

[37] V. Barger and C. Kao, Phys. Rev. D 60, 115015 (1999); K. T. Matchev and D. M. Pierce, ibid. 60, 075004 (1999); H. Baer, M. Drees, F. Paige, P. Quintana, and X. Tata, ibid. 61, 095007 (2000). 